\title{
COHERENT ADEQUATE FORCING AND PRESERVING CH
}

\author{
JOHN KRUEGER AND MIGUEL ANGEL MOTA
}

\begin{abstract}
We develop a general framework for forcing with coherent adequate sets on $H(\lambda)$ as side conditions, where $\lambda \geq \omega_{2}$ is a cardinal of uncountable cofinality. We describe a class of forcing posets which we call coherent adequate type forcings. The main theorem of the paper is that any coherent adequate type forcing preserves $\mathrm{CH}$. We show that there exists a forcing poset for adding a club subset of $\omega_{2}$ with finite conditions while preserving $\mathrm{CH}$, solving a problem of Friedman 3 .
\end{abstract}

The method of side conditions, invented by Todorčević (10]), describes a style of forcing in which elementary substructures are included in the conditions of a forcing poset to ensure that the forcing poset preserves cardinals. Friedman (3]) and Mitchell ([7]) independently took the first steps in generalizing the method from adding generic objects of size $\omega_{1}$ to adding larger objects by defining forcing posets with finite conditions for adding a club subset of $\omega_{2}$. Neeman (9]) was the first to simplify the side conditions of Friedman and Mitchell and present a generally applicable technique for forcing on $\omega_{2}$ with finite conditions.

Krueger ([5]) developed an alternative framework for forcing objects of size $\omega_{2}$ with finite conditions, using adequate sets of models as side conditions. An adequate set of models consists of countable models which are pairwise membership comparable up to some initial segment. Later Krueger ([6]) introduced the idea of coherent adequate sets, which requires the existence of isomorphisms between certain models in an adequate set. This idea combined adequate sets with an isomorphism structure originally used by Todorčević [10] in the context of forcing on $\omega_{1}$. Coherent adequate sets were applied in [6] to define a strongly proper forcing poset which forces $\square_{\omega_{1}}$.

The present paper makes advances on the framework of coherent adequate sets. We present a more general development in the context of $H(\lambda)$ for a cardinal $\lambda \geq \omega_{2}$ of uncountable cofinality, rather than just $H\left(\omega_{2}\right)$ as was treated in 6. We define a class of forcing posets which we call coherent adequate type forcings. The main theorem of the paper is that any coherent adequate type forcing preserves $\mathrm{CH}$. More generally, any coherent adequate type forcing on $H(\lambda)$, where $2^{\omega}<\lambda$ is a cardinal of uncountable cofinality, collapses $2^{\omega}$ to have size $\omega_{1}$ and forces $\mathrm{CH}$. We describe coherent adequate type forcings for adding a square sequence and for adding a club to a fat stationary subset of $\omega_{2}$.

The forcing posets of Friedman, Mitchell, and Neeman for adding a club subset of $\omega_{2}$ with finite conditions all force that $2^{\omega}=\omega_{2}$. Any forcing poset which has strongly generic conditions for countable models will add reals, including those defined in this paper. These earlier forcings for adding clubs with finite conditions

Date: June, 2014.

2010 Mathematics Subject Classification. Primary: 03E40. Secondary: 03E05.

Key words and phrases. Forcing, side conditions, adequate sets, coherent adequate sets. 
can be factored in many ways so that the quotient forcing also has strongly generic conditions in the intermediate extension. For this reason, these posets add $\omega_{2}$ many distinct reals. Friedman ([3]) asked whether it is possible to add a club subset of $\omega_{2}$ with finite conditions while preserving $\mathrm{CH}$. We solve this problem by defining a forcing poset which adds a club to a fat stationary set and falls in the class of coherent adequate type forcings.

Finally we show that, under $\mathrm{CH}$, the forcing poset consisting of finite coherent adequate subsets of $H(\lambda)$ ordered by inclusion, where $\lambda \geq \omega_{2}$ is regular, is $\omega_{2}$-c.c. and therefore preserves all cardinals.

Section 1 develops the basic ideas of adequate and coherent adequate sets. This development is almost self contained, except for three results for which we refer the reader to the corresponding results of [5] for proofs. Differences between the current paper and earlier papers on adequate sets include the consideration of a more general context, namely countable elementary substructures of $H(\lambda)$ for some $\lambda \geq \omega_{2}$ of uncountable cofinality, rather than just $H\left(\omega_{2}\right)$. Also we omit the assumption that $2^{\omega_{1}}=\omega_{2}$. Since we are not interested in taking initial segments of models as was done in [5], the set $\Lambda$ which is used to compare models can be taken to be $\omega_{2} \cap \operatorname{cof}\left(\omega_{1}\right)$. Some simplifications of the material in [5] follow from these new conventions and from the presence of isomorphisms between models.

Section 2 proves the main result of the paper, that any coherent adequate type forcing preserves $\mathrm{CH}$. Section 3 proves that if $\mathrm{CH}$ fails, then any coherent adequate type forcing on $H(\lambda)$, where $\lambda>2^{\omega}$ is a cardinal of uncountable cofinality, collapses $2^{\omega}$ to have size $\omega_{1}$, preserves $\left(2^{\omega}\right)^{+}$, and forces CH. Sections 4 and 5 develop the technical machinery for amalgamating conditions over countable elementary substructures. Sections 6 and 7 give examples of coherent adequate type forcings. In Section 6 we review the poset from [6] for adding a square sequence. In Section 7 we define a coherent adequate type forcing poset which adds a club to a fat stationary subset of $\omega_{2}$.

Section 8 presents general results for amalgamating coherent adequate sets over elementary substructures of size $\omega_{1}$. These results are not needed for the present paper, but could be useful for future applications. We show that the forcing poset consisting of finite coherent adequate subsets of $H(\lambda)$ ordered by end-extension is $\omega_{2}$-c.c.

The general development of coherent adequate sets presented in Sections 1, 4, 5, and 8 is due to Krueger.

Asperó and Mota (2] ) proved recently that for any cardinal $\lambda \geq \omega_{2}$ of uncountable cofinality, the forcing poset consisting of finite symmetric systems of countable elementary substructures of $H(\lambda)$ ordered by inclusion preserves $\mathrm{CH}$. A symmetric system is similar to a coherent adequate set, except that it does not have the adequate structure. Also Todorčević pointed out to the authors that in an unpublished result from the 1980s he proved using a different argument that forcing with finite sets of countable elementary substructures of $H\left(\omega_{2}\right)$ with isomorphisms, of the type described at the end of [10, preserves $\mathrm{CH}$ and adds an $\omega_{1}$-Kurepa tree.

Neither of these results show how to force with side conditions together with another finite set of objects to preserve $\mathrm{CH}$, nor do they imply anything regarding adequate set forcing. By arguments of Miyamoto [8, any coherent adequate type forcing on $H(\lambda)$ adds an $\omega_{1}$-tree with $\lambda$ many cofinal branches, for any regular 
cardinal $\lambda \geq \omega_{2}$. Thus $\omega_{1}$-Kurepa trees exist in coherent adequate type forcing extensions.

\section{Coherent Adequate Sets}

In this section we present the basic framework of coherent adequate sets. We will assume throughout the paper that $\lambda \geq \omega_{2}$ is a fixed cardinal of uncountable cofinality. This implies that any countable subset of $H(\lambda)$ is a member of $H(\lambda)$. We also fix a predicate $Y \subseteq H(\lambda)$, which we assume codes a well-ordering of $H(\lambda)$ among other things.

Let $\mathcal{X}$ denote the set of $N \subseteq H(\lambda)$ such that $N$ is countable and $N \prec(H(\lambda), \in$ $, Y)$. We introduce a way to compare members of $\mathcal{X}$. Fix $\Lambda$ a cofinal subset of $\omega_{2} \cap \operatorname{cof}\left(\omega_{1}\right)$.

Definition 1.1. For $M \in \mathcal{X}$, let $\Lambda_{M}$ denote the set of $\beta \in \Lambda$ such that

$$
\beta=\min (\Lambda \backslash(\sup (M \cap \beta)) .
$$

Since $M$ is countable, it has countably many limit points. It follows easily that $\Lambda_{M}$ is countable.

Lemma 1.2. Let $M \in \mathcal{X}$ and $\beta \in \Lambda_{M}$. If $\beta_{0} \in \Lambda \cap \beta$ then $M \cap\left[\beta_{0}, \beta\right) \neq \emptyset$.

Proof. Suppose for a contradiction that $M \cap\left[\beta_{0}, \beta\right)=\emptyset$. Then $\sup (M \cap \beta) \leq \beta_{0}$. So $\beta=\min (\Lambda \backslash(\sup (M \cap \beta))) \leq \beta_{0}$, which contradicts that $\beta_{0}<\beta$.

Lemma 1.3. For $M$ and $N$ in $\mathcal{X}, \Lambda_{M} \cap \Lambda_{N}$ has a largest element.

We omit the proof and refer the reader to Lemma 2.4 of [5], whose proof is nearly identical to that needed in the present context.

Definition 1.4. For $M$ and $N$ in $\mathcal{X}$, let $\beta_{M, N}$ denote the largest element of $\Lambda_{M} \cap$ $\Lambda_{N}$. The ordinal $\beta_{M, N}$ is called the comparison point of $M$ and $N$.

Given a set $K$ in $\mathcal{X}$, let $K^{\prime}$ denote the set $\left(K \cap \omega_{2}\right) \cup\left(\lim \left(K \cap \omega_{2}\right)\right)$.

Lemma 1.5. Let $M$ and $N$ be in $\mathcal{X}$. Then $M^{\prime} \cap N^{\prime} \subseteq \beta_{M, N}$.

The proof is almost the same as the proof of Proposition 2.6 of [5], so we skip it.

If $K$ and $M$ are in $\mathcal{X}$ and $K \subseteq M$, then an easy argument shows that $\Lambda_{K} \subseteq \Lambda_{M}$. It follows that for all $N$ in $\mathcal{X}, \max \left(\Lambda_{K} \cap \Lambda_{N}\right) \leq \max \left(\Lambda_{M} \cap \Lambda_{N}\right)$. So $\beta_{K, N} \leq \beta_{M, N}$.

We define relations $<, \leq$, and $\sim$ on $\mathcal{X}$. Let $M<N$ if $M \cap \beta_{M, N} \in N$. Let $M \sim N$ if $M \cap \beta_{M, N}=N \cap \beta_{M, N}$. Let $M \leq N$ if either $M<N$ or $M \sim N$.

Definition 1.6. A subset $A$ of $\mathcal{X}$ is said to be adequate if for all $M$ and $N$ in $A$, either $M<N, M \sim N$, or $N<M$.

Note that any subset of an adequate set is adequate. Also if $A$ is finite and adequate, $M \in \mathcal{X}$, and $A \in M$, then $A \cup\{M\}$ is adequate.

Suppose that $M<N$. Then $M \cap \beta_{M, N} \in N$. But $\beta_{M, N} \in \Lambda_{M}$ implies that $\beta_{M, N}=\min \left(\Lambda \backslash\left(\sup \left(M \cap \beta_{M, N}\right)\right)\right)$. Hence $\beta_{M, N}$ is definable in $H(\lambda)$ from $M \cap \beta_{M, N}$. Since $N$ is elementary in $H(\lambda), \beta_{M, N}$ is in $N$.

Lemma 1.7. Let $M$ and $N$ be in $\mathcal{X}$ and $\beta \in \Lambda$.

(1) If $M \cap \omega_{2} \subseteq \beta$, then $\beta_{M, N} \leq \beta$.

(2) If $\beta<\beta_{M, N}$ and $\{M, N\}$ is adequate, then $M \cap N \cap\left[\beta, \beta_{M, N}\right) \neq \emptyset$. 
Proof. (1) Suppose for a contradiction that $\beta<\beta_{M, N}$. Then since $\beta \in \Lambda$ and $\beta_{M, N} \in \Lambda_{M}, M \cap\left[\beta, \beta_{M, N}\right) \neq \emptyset$ by Lemma 1.2. This contradicts that $M \cap \omega_{2} \subseteq \beta$.

(2) Without loss of generality assume that $M \leq N$. Then $M \cap \beta_{M, N} \subseteq N$. By Lemma 1.2, fix $\xi$ in $M \cap\left[\beta, \beta_{M, N}\right)$. Then $\xi$ is in $M \cap \beta_{M, N}$ and hence in $N$. So $\xi$ is in $M \cap N \cap\left[\beta, \beta_{M, N}\right)$.

Next we define remainder points, which describe the overlap of models past their comparison point.

Definition 1.8. Let $M$ and $N$ be in $\mathcal{X}$ and assume that $\{M, N\}$ is adequate. Define $R_{M}(N)$ as the set of $\beta$ satisfying either:

(1) $N \leq M$ and $\beta=\min \left(N \backslash \beta_{M, N}\right)$, or

(2) there is $\gamma \in M \backslash \beta_{M, N}$ such that $\beta=\min (N \backslash \gamma)$.

The set $R_{M}(N)$ is called the set of remainder points of $N$ over $M$. This set is always finite, since otherwise there would be a common limit point of $M$ and $N$ greater than $\beta_{M, N}$, contradicting Lemma 1.5. For a more detailed proof, see Proposition 2.9 of [5].

Suppose that $M<N$. Then by definition, if $\zeta \in R_{M}(N)$ then there is $\gamma \in$ $M \backslash \beta_{M, N}$ such that $\zeta=\min (N \backslash \gamma)$. On the other hand, consider $\zeta \in R_{N}(M)$. Since $\beta_{M, N}$ is in $N$ as noted above, if $\zeta=\min \left(M \backslash \beta_{M, N}\right)$, then again there is $\gamma \in N \backslash \beta_{M, N}$ such that $\zeta=\min (M \backslash \gamma)$, namely $\gamma=\beta_{M, N}$. So remainder points of models $M$ and $N$ are given just by condition (2) in Definition 1.8 in the case that $M<N$ or $N<M$. Condition (1) is only relevant when $M \cap \beta_{M, N}=N \cap \beta_{M, N}$.

Given an adequate set $A$, define $R_{A}$ by letting

$$
R_{A}=\bigcup\left\{R_{N}(M): M, N \in A\right\} .
$$

Definition 1.9. For a given set $S \subseteq \omega_{2}$, a set $A \subseteq \mathcal{X}$ is $(S)$ adequate if it is adequate and $R_{A} \subseteq S$.

For the rest of the paper we let $\Lambda:=\omega_{2} \cap \operatorname{cof}\left(\omega_{1}\right)$. Note that $\Lambda$ is a definable subset of $H(\lambda)$.

Now we move on to coherent adequate sets. We will consider isomorphisms between models in $\mathcal{X}$.

Let $M$ and $N$ be in $\mathcal{X}$ and let $\sigma: M \rightarrow N$. We say that $\sigma$ is an isomorphism if $\sigma$ is a bijection and for all $a$ and $b$ in $M$,

- $a \in b$ iff $\sigma(a) \in \sigma(b)$;

- $a \in Y$ iff $\sigma(a) \in Y$.

In other words, $\sigma$ is an isomorphism if it is an isomorphism in the usual model theoretic sense between the structures $(M, \in, Y \cap M)$ and $(N, \in, Y \cap N)$. We say that $M$ and $N$ are isomorphic if there exists an isomorphism from $M$ to $N$. Note that the isomorphism relation is an equivalence relation.

For a model $M$ in $\mathcal{X}$, the elementarity of $M$ in $H(\lambda)$ implies that $M$ satisfies the axiom of extensionality. It follows that $(M, \in, Y \cap M)$ is isomorphic to a unique transitive structure $(\bar{M}, \in, \bar{Y})$ by a unique isomorphism $\sigma_{M}: M \rightarrow \bar{M}$ given by the recursive equation $\sigma_{M}(a)=\sigma_{M}[a \cap M]$. Note that if $a \in M$ is countable, then $a \subseteq M$, so $\sigma_{M}(a)=\sigma_{M}[a]$.

By the uniqueness of the transitive collapse, a standard argument shows that $M$ and $N$ in $\mathcal{X}$ are isomorphic iff the structures $(M, \in, Y \cap M)$ and $(N, \in, Y \cap N)$ have 
the same transitive collapse. In that case, $\sigma_{N}^{-1} \circ \sigma_{M}$ is an isomorphism from $M$ to $N$, which we denote by $\sigma_{M, N}$. Also the uniqueness of the transitive collapsing map easily implies that $\sigma_{M, N}$ is the unique isomorphism from $M$ to $N$. Note that this map satisfies that if $a \in M$ is countable, then $\sigma_{M, N}(a)=\sigma_{M, N}[a]$. Also by the uniqueness of isomorphisms, if $M, N$, and $P$ are isomorphic, then $\sigma_{M, P}=$ $\sigma_{N, P} \circ \sigma_{M, N}$.

Lemma 1.10. Let $M, N$ and $K$ be in $\mathcal{X}$ such that $M$ and $N$ are isomorphic and $K \in M$. Let $L:=\sigma_{M, N}(K)$. Then:

(1) $L \in \mathcal{X}$;

(2) $K$ and $L$ are isomorphic and $\sigma_{K, L}=\sigma_{M, N} \uparrow K$.

Proof. (1) As $L \subseteq N$ and $(N, \in, N \cap Y) \prec(H(\lambda), \in, Y)$, it suffices to show that $\mathfrak{L}:=(L, \in, L \cap Y) \prec(N, \in, N \cap Y)$. Let $\mathfrak{K}:=(K, \in, M \cap Y)$. By the elementarity of $M$ and $N$, the predicates $K \cap Y$ are $L \cap Y$ are in $M$ and $N$ respectively. Let $b_{1}, \ldots, b_{k}$ be in $L$ and let $\varphi\left(x_{1}, \ldots, x_{k}\right)$ be a formula in the language of the structure $(H(\lambda), \in, Y)$. Let $a_{i}:=\sigma_{N, M}\left(b_{i}\right)$ for $i=1, \ldots, k$. Then $(L, \in, L \cap Y) \models \varphi\left[b_{1}, \ldots, b_{k}\right]$ iff $N \models\ulcorner\varphi\urcorner^{\mathfrak{L}}\left[b_{1}, \ldots, b_{k}\right]$ iff $M \models\ulcorner\varphi\urcorner^{\mathfrak{K}}\left[a_{1}, \ldots, a_{k}\right]$ iff $M \models \varphi\left[a_{1}, \ldots, a_{k}\right]$ iff $N \models \varphi\left[b_{1}, \ldots, b_{k}\right]$, where the third equivalence follows from the fact that $\mathfrak{K}$ is an elementary substructure of $(M, \in, M \cap Y)$.

(2) Since $K$ is countable, $\sigma_{M, N}(K)=\sigma_{M, N}[K]$. So $\sigma_{M, N} \uparrow K$ is a bijection from $K$ to $L$. It is obvious that $\sigma_{M, N} \uparrow K$ preserves the predicates $\in$ and $Y$ since $\sigma_{M, N}$ does. So $\sigma_{M, N} \uparrow K$ is an isomorphism of $K$ to $L$. By the uniqueness of isomorphisms, $\sigma_{M, N} \uparrow K=\sigma_{K, L}$.

Lemma 1.11. Suppose that $M$ and $N$ are in $\mathcal{X}$ and are isomorphic. Let $\sigma:=\sigma_{M, N}$. Assume that $K$ and $L$ are in $M \cap \mathcal{X}$. Then:

(1) $\sigma\left(\beta_{K, L}\right)=\beta_{\sigma(K), \sigma(L)}$;

(2) $K<L$ iff $\sigma(K)<\sigma(L)$;

(3) $K \sim L$ iff $\sigma(K) \sim \sigma(L)$;

(4) $K \leq L$ iff $\sigma(K) \leq \sigma(L)$;

(5) $\sigma\left(R_{K}(L)\right)=R_{\sigma(K)}(\sigma(L))$;

(6) if $\{K, L\}$ is adequate then $\{\sigma(K), \sigma(L)\}$ is adequate.

Proof. The lemma follows from the fact for any $P$ and $Q$ in $\mathcal{X}$, the objects and relations $\beta_{P, Q}, P<Q, P \leq Q$, and $R_{P}(Q)$ are definable in $H(\lambda)$ from $P$ and $Q$.

We now introduce an additional requirement on isomorphisms. Let us say that $M$ and $N$ in $\mathcal{X}$ are strongly isomorphic if they are isomorphic and for all $a \in M \cap N$, $\sigma_{M, N}(a)=a$. We write $M \cong N$ to indicate that $M$ and $N$ are strongly isomorphic.

Lemma 1.12. Let $M, N$ and $K$ be in $\mathcal{X}$ such that $M$ and $N$ are strongly isomorphic and $K \in M$. Let $L:=\sigma_{M, N}(K)$. Then $K$ and $L$ are strongly isomorphic.

Proof. By Lemma 1.10, $K$ and $L$ are isomorphic and $\sigma_{K, L}=\sigma_{M, N} \uparrow K$. Let $a \in K \cap L$ be given. Then $a \in M \cap N$. So $\sigma_{K, L}(a)=\sigma_{M, N}(a)=a$.

Lemma 1.13. Suppose that $N, N^{\prime}$, and $N^{*}$ are in $\mathcal{X}$ and are strongly isomorphic. Let $a \in N^{\prime} \cap N^{*}$. Then $\sigma_{N^{\prime}, N}(a)=\sigma_{N^{*}, N}(a)$.

Proof. Since $a \in N^{\prime} \cap N^{*}, \sigma_{N^{\prime}, N^{*}}(a)=a$. So $\sigma_{N^{\prime}, N}(a)=\sigma_{N^{*}, N}\left(\sigma_{N^{\prime}, N^{*}}(a)\right)=$ $\sigma_{N^{*}, N}(a)$. 
Lemma 1.14. Let $N, N^{\prime}, N^{*}, K$, and $L$ be in $\mathcal{X}$ such that $N, N^{\prime}$, and $N^{*}$ are strongly isomorphic, $K$ and $L$ are strongly isomorphic, and $K$ and $L$ are in $N$. Let $M:=\sigma_{N, N^{\prime}}(K)$ and $P:=\sigma_{N, N^{*}}(L)$. Then $M$ and $P$ are strongly isomorphic.

Proof. By Lemma 1.10, $K$ and $M$ are isomorphic and $L$ and $P$ are isomorphic. Since $K$ and $L$ are isomorphic and being isomorphic is an equivalence relation, $M$ and $P$ are isomorphic. To show that $M$ and $P$ are strongly isomorphic, let $a \in M \cap P$ and we will show that $\sigma_{M, P}(a)=a$. Let $b:=\sigma_{N^{\prime}, N}(a)$. Since $a \in M \cap P, a \in N^{\prime} \cap N^{*}$. By Lemma 1.13, $\sigma_{N^{*}, N}(a)=\sigma_{N^{\prime}, N}(a)=b$. Now $a \in M$ implies that $b \in K$, and $a \in P$ implies that $b \in L$. So $b \in K \cap L$. Since $K$ and $L$ are strongly isomorphic, $\sigma_{K, L}(b)=b$. So $\sigma_{M, P}(a)=\sigma_{L, P}\left(\sigma_{K, L}\left(\sigma_{M, K}(a)\right)\right)=\sigma_{N, N^{*}}\left(\sigma_{K, L}\left(\sigma_{N^{\prime}, N}(a)\right)\right)=$ $\sigma_{N, N^{*}}\left(\sigma_{K, L}(b)\right)=\sigma_{N, N^{*}}(b)=a$.

Definition 1.15. Let $A$ be a subset of $\mathcal{X}$. We say that $A$ is coherent adequate if $A$ is adequate, and for all $M$ and $N$ in $A$ :

(1) if $M \sim N$, then $M$ and $N$ are strongly isomorphic;

(2) if $M<N$, then there exists $N^{\prime} \in A$ isomorphic to $N$ such that $M \in N^{\prime}$;

(3) if $M$ and $N$ are isomorphic and $K \in M \cap A$, then $\sigma_{M, N}(K) \in A$.

Let $M$ and $N$ be in $\mathcal{X}$. Since $\omega_{1} \leq \beta_{M, N}, M \sim N$ implies that $M \cap \omega_{1}=N \cap \omega_{1}$. Also if $M<N$, then $M \cap \omega_{1}$ is an initial segment of $M \cap \beta_{M, N}$ and hence is in $N$. It follows that if $\{M, N\}$ is adequate, then $M \sim N$ iff $M \cap \omega_{1}=N \cap \omega_{1}$. Also if $M$ and $N$ are isomorphic, then since $\omega_{1}$ is definable in $H(\lambda), \sigma_{M, N}\left(\omega_{1}\right)=\omega_{1}$. It easily follows that $M \cap \omega_{1}=N \cap \omega_{1}$.

As a consequence of these observations, if $A$ is coherent adequate and $M$ and $N$ are in $A$, then the following are equivalent:

- $M \sim N$

- $M \cap \omega_{1}=N \cap \omega_{1}$

- $M$ and $N$ are isomorphic;

- $M$ and $N$ are strongly isomorphic.

Moreover, $M<N$ iff $M \cap \omega_{1}<N \cap \omega_{1}$.

Given a set $S \subseteq \omega_{2}$, a set $A \subseteq \mathcal{X}$ is $(S)$ coherent adequate if $A$ is coherent adequate and $R_{A} \subseteq S$.

Lemma 1.16. Let $A$ be a finite coherent adequate set.

(1) If $M \in \mathcal{X}$ and $A \in M$, then $A \cup\{M\}$ is coherent adequate.

(2) $M \cap A$ is coherent adequate.

Proof. (1) We already observed that $A \cup\{M\}$ is adequate, and the coherent properties are immediate. (2) Requirements (1) and (3) in the definition of coherent adequate are obvious. For requirement (2), suppose that $K$ and $N$ are in $A \cap M$ and $K<N$. Since $A$ is coherent, there is $N^{\prime} \in A$ isomorphic to $N$ such that $K \in N^{\prime}$. If $N^{\prime} \in M$ then we are done, so assume not. As $N^{\prime} \cap \omega_{1}=N \cap \omega_{1}<M \cap \omega_{1}$, $N^{\prime}<M$. So there is $M^{\prime} \in A$ isomorphic to $M$ with $N^{\prime} \in M^{\prime}$. Let $N^{*}=\sigma_{M^{\prime}, M}\left(N^{\prime}\right)$, which is in $M \cap A$. Since $K \in N^{\prime}$ and $N^{\prime} \in M^{\prime}, K \in M^{\prime}$. But also $K \in M$, so $\sigma_{M^{\prime}, M}(K)=K$. Since $K \in N^{\prime}, K=\sigma_{M^{\prime}, M}(K) \in \sigma_{M, M^{\prime}}\left(N^{\prime}\right)=N^{*}$. So $K \in N^{*}$, $N^{*}$ is isomorphic to $N$, and $N^{*} \in M \cap A$.

Lemma 1.17. Let $A$ be a finite coherent adequate set. Suppose that $\left\{M_{0}, \ldots, M_{k}\right\}$ is adequate and consists of strongly isomorphic sets. Assume that $A \in M_{0} \cap \cdots \cap M_{k}$. Then $A \cup\left\{M_{0}, \ldots, M_{k}\right\}$ is coherent adequate. 
Proof. It is obvious that $A \cup\left\{M_{0}, \ldots, M_{k}\right\}$ is adequate and satisfies requirements (1) and (2) in the definition of coherent. Requirement (3) follows from the fact that for any $i, j \leq k$, if $K \in M_{i} \cap A$, then $K \in M_{j}$, and therefore $\sigma_{M_{i}, M_{j}}(K)=K \in A$.

Lemma 1.18. Let $M$ and $N$ be in $\mathcal{X}$ and assume that $M$ and $N$ are strongly isomorphic. Then $M \cap P_{\omega_{1}}(M \cap N) \subseteq N$. In particular, if $\{M, N\}$ is adequate and $M$ and $N$ are strongly isomorphic, then $M \cap P_{\omega_{1}}\left(\beta_{M, N}\right) \subseteq N$.

Proof. Let $a \in M \cap P_{\omega_{1}}(M \cap N)$ be given. Since $a$ is countable and $\sigma_{M, N}$ is the identity on $M \cap N, \sigma_{M, N}(a)=\sigma_{M, N}[a]=a$. So $a \in N$. If in addition we know that $\{M, N\}$ is adequate, then $M \sim N$. So if $a \in M \cap P_{\omega_{1}}\left(\beta_{M, N}\right)$, then $a$ is a countable subset of $M \cap \beta_{M, N}=N \cap \beta_{M, N}$. Hence $a \in M \cap P_{\omega_{1}}(M \cap N)$ and therefore $a \in N$.

Lemma 1.19. Let $A$ be a coherent adequate set. Suppose that $M$ and $N$ are in $A$ and $M \leq N$. Then $M \cap P_{\omega_{1}}\left(\beta_{M, N}\right) \subseteq N$.

Proof. If $M$ and $N$ are strongly isomorphic, then we are done by the preceding lemma. Assume that $M<N$. Since $A$ is coherent, fix $N^{\prime} \in A$ isomorphic to $N$ such that $M \in N^{\prime}$. Then $N$ and $N^{\prime}$ are strongly isomorphic. Consider $a \in$ $M \cap P_{\omega_{1}}\left(\beta_{M, N}\right)$. Then $a \in N^{\prime} \cap P_{\omega_{1}}\left(\beta_{M, N}\right)$. Since $M \in N^{\prime}, \beta_{M, N} \leq \beta_{N, N^{\prime}}$. So $a \in N^{\prime} \cap P_{\omega_{1}}\left(\beta_{N^{\prime}, N}\right)$. As $N$ and $N^{\prime}$ are strongly isomorphic, $a \in N$ by the preceding lemma.

\section{Preserving $\mathrm{CH}$}

Fix for the remainder of this section a set $S \subseteq \omega_{2}$ such that $S \cap \operatorname{cof}\left(\omega_{1}\right)$ is stationary and a set $\mathcal{Y} \subseteq \mathcal{X}$ which is stationary in $P_{\omega_{1}}(H(\lambda))$. Also assume that $\mathcal{Y}$ is closed under isomorphisms, which means that whenever $M$ and $N$ are in $\mathcal{Y}$ and are isomorphic, and $K \in M \cap \mathcal{Y}$, then $\sigma_{M, N}(K) \in \mathcal{Y}$. Note that by Lemma 1.10, the set $\mathcal{X}$ itself is closed under isomorphisms.

A forcing poset $\mathbb{P}$ is said to be an $(S, \mathcal{Y})$ coherent adequate type forcing if there exists a natural number $m$ such that $\mathbb{P}$ consists of conditions of the form $\left(x_{0}, \ldots, x_{m}, A\right)$ satisfying:

(I) $x_{0}, \ldots, x_{m}$ are finite subsets of $H(\lambda)$;

(II) $A$ is a finite $(S)$ coherent adequate subset of $\mathcal{Y}$;

(III) if $\left(y_{0}, \ldots, y_{m}, B\right) \leq\left(x_{0}, \ldots, x_{m}, A\right), N$ and $N^{\prime}$ are isomorphic sets in $B$, and $\left(x_{0}, \ldots, x_{m}, A\right) \in N$, then $\sigma_{N, N^{\prime}}\left(\left(x_{0}, \ldots, x_{m}, A\right)\right)$ is in $\mathbb{P}$, and $\left(y_{0}, \ldots, y_{m}, B\right) \leq \sigma_{N, N^{\prime}}\left(\left(x_{0}, \ldots, x_{m}, A\right)\right)$;

(IV) if $M_{0}, \ldots, M_{n}$ are isomorphic sets in $\mathcal{Y}$ such that $\left\{M_{0}, \ldots, M_{n}\right\}$ is $(S)$ coherent adequate and $\left(x_{0}, \ldots, x_{m}, A\right) \in M_{0} \cap \cdots \cap M_{n}$, then there is a condition $\left(y_{0}, \ldots, y_{m}, B\right) \leq\left(x_{0}, \ldots, x_{m}, A\right)$ such that $M_{0}, \ldots, M_{n} \in B$

(V) for all $M \in A,\left(x_{0}, \ldots, x_{m}, A\right)$ is strongly $M$-generic.

Regarding $(\mathrm{V})$, recall that a condition $q$ is strongly $M$-generic if for any set $D$ which is dense in the forcing poset $M \cap \mathbb{P}, D$ is predense in $\mathbb{P}$ below $q$.

We say that $\mathbb{P}$ is a coherent adequate type forcing if $\mathbb{P}$ is an $\left(\omega_{2}, \mathcal{X}\right)$ coherent type forcing. Define $(S)$ coherent adequate and $(\mathcal{Y})$ coherent adequate type forcings similarly. We interpret the above definition to include the possibility that the sequence $x_{0}, \ldots, x_{m}$ in a condition has length 0 , in which case conditions are just $(S, \mathcal{Y})$ coherent adequate sets.

Lemma 2.1. Let $\mathbb{P}$ be an $(S, \mathcal{Y})$ coherent adequate forcing. Then $\mathbb{P}$ preserves $\omega_{1}$. 
Proof. Let $\dot{f}$ be a $\mathbb{P}$-name for a function from $\omega$ to $\omega_{1}$ and let $p$ be a condition. We will find $q \leq p$ which forces that the range of $\dot{f}$ is bounded. Fix $\chi \geq \lambda$ regular such that $\dot{f} \in H(\chi)$. Since $\mathcal{Y}$ is stationary, we can find $N^{*}$ a countable elementary substructure of $H(\chi)$ with $\mathbb{P}, \dot{f}$, and $p$ in $N^{*}$ and $N:=N^{*} \cap H(\lambda)$ in $\mathcal{Y}$.

By property (IV), there is $q=\left(y_{0}, \ldots, y_{m}, B\right) \leq p$ such that $N \in B$. By property $(\mathrm{V}), q$ is strongly $N$-generic. It is easy to see that for all $n<\omega$, the set of $s \in N \cap \mathbb{P}$ which decide the value of $\dot{f}(n)$ is dense in $N \cap \mathbb{P}$. It follows that for all $n<\omega$, if $r \leq q$ decides the value of $\dot{f}(n)$, then that value is a member of $N$. So $q$ forces that $\dot{f}$ is bounded.

We will prove that any $(S, \mathcal{Y})$ coherent adequate type forcing preserves $\omega_{2}$ and $\mathrm{CH}$. The proof will use the following technical result.

Lemma 2.2. (CH) Suppose that $R_{0}, \ldots, R_{k}$ are subsets of $H(\lambda)$. Then for any set $z \in H(\lambda)$, there are $M$ and $N$ in $\mathcal{Y}$ satisfying the following:

(1) $z \in M \cap N$;

(2) $\{M, N\}$ is $(S)$ coherent adequate;

(3) the structures $\left(M, \in, Y \cap M, R_{0} \cap M, \ldots, R_{k} \cap M\right)$ and $\left(N, \in, Y \cap N, R_{0} \cap\right.$ $\left.N, \ldots, R_{k} \cap N\right)$ are elementary in $\left(H(\lambda), \in, Y, R_{0}, \ldots, R_{k}\right)$ and are isomorphic;

(4) $M \cap \omega_{2} \subseteq \min \left(N \backslash \beta_{M, N}\right)$ and $\sigma_{M, N}\left(\min \left(M \backslash \beta_{M, N}\right)\right)=\min \left(N \backslash \beta_{M, N}\right)$.

Proof. Since $S \cap \operatorname{cof}\left(\omega_{1}\right)$ is stationary and CH holds, we can fix $N^{*}$ satisfying:

(1) $N^{*} \prec H\left(2^{\lambda}\right)$;

(2) $\left|N^{*}\right|=\omega_{1}$;

(3) $Y, z, S, \mathcal{Y}, R_{0}, \ldots, R_{k}$ are in $N^{*}$;

(4) $\beta^{*}:=N^{*} \cap \omega_{2} \in S$

(5) $\left(N^{*}\right)^{\omega} \subseteq N^{*}$;

As $\mathcal{Y}$ is stationary, we can fix $N$ in $\mathcal{Y}$ such that $z, \beta^{*} \in N$ and

$$
\left(N, \in, Y \cap N, R_{0} \cap N, \ldots, R_{k} \cap N\right) \prec\left(H(\lambda), \in, Y, R_{0}, \ldots, R_{k}\right) .
$$

Let $\mathfrak{N}$ denote the structure $\left(N, \in, Y \cap N, R_{0} \cap N, \ldots, R_{k} \cap N\right)$, let $\overline{\mathfrak{N}}$ denote its transitive collapse, and as usual let $\sigma_{N}$ denote the transitive collapsing map. Let $T$ be the relation defined by letting $T(a, b)$ hold if $a \in N \cap N^{*}$ and $\sigma_{N}(a)=b$. By $\mathrm{CH}, H\left(\omega_{1}\right)$ has size $\omega_{1}$. Since $\left(N^{*}\right)^{\omega} \subseteq N^{*}, H\left(\omega_{1}\right)$ is a subset of $N^{*}$. It follows that $\overline{\mathfrak{N}}$ is a member and a subset of $N^{*}$. Also $N^{*}$ contains the sets $N \cap N^{*}$ and $N \cap \beta^{*}$ as members. It follows that the relation $T$ is a subset of $N^{*}$, and since it is countable it is a member of $N^{*}$.

The objects $N$ and $\beta^{*}$ witness the statement, satisfied by the structure $H\left(2^{\lambda}\right)$, that there exist $M$ and $\beta$ satisfying:

(a) $M$ is in $\mathcal{Y}$;

(b) $\beta \in S \cap \operatorname{cof}\left(\omega_{1}\right)$;

(c) $z$ and $\beta$ are in $M$;

(d) $N \cap \beta^{*}=M \cap \beta$;

(e) $N \cap N^{*} \subseteq M$;

(f) $\left(M, \in, Y, R_{0} \cap M, \ldots, R_{k} \cap M\right) \prec\left(H(\lambda), \in, Y, R_{0}, \ldots, R_{k}\right)$;

(g) the transitive collapse of the structure $\left(M, \in, Y \cap M, R_{0} \cap M, \ldots, R_{k} \cap M\right)$ is equal to $\overline{\mathfrak{N}}$;

(h) $T(a, b)$ implies that $\sigma_{M}(a)=b$. 
Since the parameters which appear in the above statement are all members of $N^{*}$, by elementarity we can fix $M$ and $\beta$ in $N^{*}$ satisfying the same statement.

Let us prove that $M$ and $N$ are as desired. We know that $M$ and $N$ are in $\mathcal{Y}$ and $z \in M \cap N$. Also the structures $\left(M, \in, Y \cap M, R_{0} \cap M, \ldots, R_{k} \cap M\right)$ and $\left(N, \in, Y \cap N, R_{0} \cap N, \ldots, R_{k} \cap N\right)$ are elementary in $\left(H(\lambda), \in, Y, R_{0}, \ldots, R_{k}\right)$. And since they have the same transitive collapse, they are isomorphic.

We claim that $M$ and $N$ are strongly isomorphic. So let $a \in M \cap N$ be given, and we show that $\sigma_{N, M}(a)=a$. Since $M \in N^{*}$, it follows that $a \in N \cap N^{*}$. Let $b:=\sigma_{N}(a)$. Then $T(a, b)$ holds. By (h), $\sigma_{M}(a)=b$. It follows that $\sigma_{N, M}(a)=$ $\sigma_{M}^{-1}\left(\sigma_{N}(a)\right)=\sigma_{M}^{-1}(b)=a$.

We claim that $\beta_{M, N} \leq \beta$. Since $M \in N^{*}, \Lambda_{M} \subseteq N^{*}$. But $\beta_{M, N} \in \Lambda_{M}$, so $\beta_{M, N} \in N^{*} \cap \omega_{2}=\beta^{*}$. This shows that $\beta_{M, N} \leq \beta^{*}$. Let $\xi:=\sup \left(N \cap \beta_{M, N}\right)$. As $\beta_{M, N} \leq \beta^{*}, \xi$ is a limit point of $N \cap \beta^{*}$. Since $\beta_{M, N} \in \Lambda_{N}, \beta_{M, N}=\min (\Lambda \backslash \xi)$. Now $N \cap \beta^{*}=M \cap \beta$ implies that $N \cap \beta^{*} \subseteq \beta$. Since $\xi$ is a limit point of $N \cap \beta^{*}$, $\xi<\beta$. As $\beta_{M, N}=\min (\Lambda \backslash \xi)$ and $\beta \in \Lambda, \beta_{M, N} \leq \beta$.

Now $M \cap \beta=N \cap \beta^{*}$ and $\beta_{M, N} \leq \beta$ imply that

(i) $M \sim N$;

(ii) $\min \left(M \backslash \beta_{M, N}\right) \geq \beta$;

(iii) $\min \left(N \backslash \beta_{M, N}\right) \geq \beta^{*}$.

Statement (i) is immediate. For (ii), if $\tau \in M \backslash \beta_{M, N}$, then $\tau \in M \backslash N$. As $M \cap \beta \subseteq N, \tau \geq \beta$. The proof of (iii) is similar.

By (i), $\{M, N\}$ is adequate. And since $M$ and $N$ are strongly isomorphic, $\{M, N\}$ is coherent adequate. As $\beta \in M$ and $\beta^{*} \in N$, (ii) and (iii) imply that $\min (M \backslash$ $\left.\beta_{M, N}\right)=\beta$ and $\min \left(N \backslash \beta_{M, N}\right)=\beta^{*}$. Also $M \in N^{*}$ implies that $M \cap \omega_{2} \subseteq \beta^{*}$. This easily implies that $R_{M}(N)=\left\{\beta^{*}\right\}$ and $R_{N}(M)=\{\beta\}$. As $\beta$ and $\beta^{*}$ are in $S$, $\{M, N\}$ is $(S)$ coherent adequate.

Since $\beta$ and $\beta^{*}$ are the first elements of $M$ and $N$ above their common intersection, clearly $\sigma_{M, N}(\beta)=\beta^{*}$. Hence $\sigma_{M, N}\left(\min \left(M \backslash \beta_{M, N}\right)\right)=\min \left(N \backslash \beta_{M, N}\right)$. Finally, $M \cap \omega_{2} \subseteq \beta^{*}=\min \left(N \backslash \beta_{M, N}\right)$.

Proposition 2.3. (CH) Let $\mathbb{P}$ be an $(S, \mathcal{Y})$ coherent adequate type forcing. If $p$ is a condition which forces that $\left\langle\dot{f}_{i}: i<\omega_{2}^{V}\right\rangle$ is a sequence of functions from $\omega$ to $\omega$, then there is $q \leq p$ and $i<j$ such that $q$ forces that $\dot{f}_{i}=\dot{f}_{j}$.

Proof. Define a relation $R$ on $H(\lambda)$ by letting $R(z, i, n, m)$ if $z \in \mathbb{P}$ and $z \Vdash_{\mathbb{P}} \dot{f}_{i}(n)=$ $m$. By Lemma 2.2, we can fix $M$ and $N$ in $\mathcal{Y}$ satisfying:

(1) $p \in M \cap N$;

(2) $\{M, N\}$ is $(S)$ coherent adequate;

(3) the structures $(M, \in, Y \cap M, R \cap M)$ and $(N, \in, Y \cap N, R \cap N)$ are elementary in $(H(\lambda), \in, Y, R)$ and are isomorphic;

(4) $M \cap \omega_{2} \subseteq \min \left(N \backslash \beta_{M, N}\right)$ and $\sigma_{M, N}\left(\min \left(M \backslash \beta_{M, N}\right)\right)=\min \left(N \backslash \beta_{M, N}\right)$.

Let $\sigma:=\sigma_{M, N}$. By the uniqueness of isomorphisms, $\sigma$ is an isomorphism between the structures described in (3) above. Let $i:=\min \left(M \backslash \beta_{M, N}\right)$ and $j:=\min (N \backslash$ $\left.\beta_{M, N}\right)$. Then $i<j$ and $\sigma(i)=j$.

We claim that for all $z \in M \cap \mathbb{P}$ and integers $n$ and $m$, if $z \Vdash_{\mathbb{P}} \dot{f}_{i}(n)=m$ then $\sigma(z) \Vdash_{\mathbb{P}} \dot{f}_{j}(n)=m$. For assume that $z \Vdash_{\mathbb{P}} \dot{f}_{i}(n)=m$. Then $R(z, i, n, m)$ holds. Since $z, i, n$, and $m$ are in $M, \sigma(i)=j$, and $\sigma$ is an isomorphism, $R(\sigma(z), j, n, m)$ holds. By definition of $R$, this means that $\sigma(z) \Vdash_{\mathbb{P}} \dot{f}_{j}(n)=m$. 
Since $M$ and $N$ are isomorphic sets in $\mathcal{Y},\{M, N\}$ is $(S)$ coherent adequate, and $p \in M \cap N$, by property (IV) in the definition of an $(S, \mathcal{Y})$ coherent adequate type forcing, there is a condition $q=(y, B)$ below $p$ such that $M, N \in B$. By property $(\mathrm{V}), q$ is strongly $M$-generic.

We claim that $q$ forces that $\dot{f}_{i}=\dot{f}_{j}$, which completes the proof. So assume for a contradiction that there exists $r \leq q$ and $n<\omega$ such that $r \Vdash_{\mathbb{P}} \dot{f}_{i}(n) \neq \dot{f}_{j}(n)$.

Let $D$ be the set of conditions $w$ in $\mathbb{P} \cap M$ such that for some $m, w \Vdash_{\mathbb{P}} \dot{f}_{i}(n)=m$. We claim that $D$ is dense in $\mathbb{P} \cap M$. So let $v \in \mathbb{P} \cap M$ be given. Clearly there exists $w$ and $m$ such that $w \leq v$ and $w \Vdash_{\mathbb{P}} \dot{f}_{i}(n)=m$, and hence $R(w, i, n, m)$ holds. Since $v, i$, and $n$ are in $M$ and $M$ is elementary in $(H(\lambda), \in, Y, R)$, there is $w \leq v$ in $\mathbb{P} \cap M$ and $m$ such that $R(w, i, n, m)$ holds. Then $w$ is an extension of $v$ in $D$.

As $q$ is strongly $M$-generic, $D$ is predense below $q$. In particular, there is $w \in D$ such that $w$ is compatible with $r$. Fix $s \leq w, r$. So $s \leq w, M$ and $N$ are isomorphic models in $A_{s}$, and $w \in M$. By Property (III) in the definition of an $(S, \mathcal{Y})$ coherent adequate type forcing, $s \leq \sigma(w)$. Since $w \Vdash_{\mathbb{P}} \dot{f}_{i}(n)=m, \sigma(w) \Vdash_{\mathbb{P}} \dot{f}_{j}(n)=m$ by the claim above. As $s$ extends both $w$ and $\sigma(w), s$ forces that $\dot{f}_{i}(n)=\dot{f}_{j}(n)$. But this contradicts that $s \leq r$, since $r$ forces that $\dot{f}_{i}(n) \neq \dot{f}_{j}(n)$.

Corollary 2.4. (CH) Let $\mathbb{P}$ be an $(S, \mathcal{Y})$ coherent adequate type forcing. Then $\mathbb{P}$ preserves $\omega_{2}$ and $\mathrm{CH}$.

Proof. The statement that $\mathbb{P}$ preserves $C H$ is immediate from the proposition. Suppose for a contradiction that $\mathbb{P}$ does not preserve $\omega_{2}$, and let $p$ be a condition which forces that $\left|\omega_{2}^{V}\right|=\omega_{1}$. Then we can find a sequence of names which $p$ forces is an enumeration of $\omega_{1}$ many distinct functions from $\omega$ to $\omega$ in order type $\omega_{2}^{V}$, contradicting the proposition.

\section{Collapsing the Continuum}

In this section we analyze what happens when we force with a coherent adequate type forcing over a model in which $\mathrm{CH}$ fails. We will prove that in this context, the cardinal $\left(2^{\omega}\right)^{V}$ will be collapsed to have size $\omega_{1}$, its successor in $V$ will become $\omega_{2}$, and $\mathrm{CH}$ will hold in the extension .

Let $\lambda$ be any cardinal with uncountable cofinality such that $2^{\omega}<\lambda$. Let $\left\langle r_{i}: i<\right.$ $\left.2^{\omega}\right\rangle$ be an enumeration of the power set of $\omega$ such that $r_{i} \neq r_{j}$ for all $i<j<2^{\omega}$. Moreover, assume that $\left\langle r_{i}: i\left\langle 2^{\omega}\right\rangle\right.$ is the first such enumeration according to the well-ordering of $H(\lambda)$ coded by the predicate $Y$. It follows that $Y$ codes the relation $Z$, where $Z(i, n)$ holds if $i<2^{\omega}$ and $n \in r_{i}$.

Lemma 3.1. Suppose that $M$ and $N$ are in $\mathcal{X}$ and are isomorphic. Then $\sigma_{M, N}(\alpha)=$ $\alpha$ for all $\alpha \in M \cap 2^{\omega}$. Therefore $M \cap 2^{\omega}=N \cap 2^{\omega}$.

Proof. Let $\alpha \in M \cap 2^{\omega}$ be given. Since $r_{i} \neq r_{j}$ for all $i<j<2^{\omega}$, it suffices to show that $r_{\alpha}=r_{\sigma_{M, N}(\alpha)}$. So let $n<\omega$ be given. Then $n \in r_{\alpha}$ iff $M \models Z(\alpha, n)$ iff $N \models Z\left(\sigma_{M, N}(\alpha), n\right)$ iff $n \in r_{\sigma_{M, N}}(\alpha)$.

Theorem 3.2. Let $\mathbb{P}$ be an $(S, \mathcal{Y})$ coherent adequate type forcing. Let $\mu$ be the cardinal $2^{\omega}$. Then $\mathbb{P}$ collapses $\mu$ to have size $\omega_{1}$.

Proof. Suppose that $p=\left(x_{0}, \ldots, x_{k}, A\right)$ is a condition, $M$ and $N$ are in $A$, and $M<N$. We claim that $M \cap \mu \in N$. By coherence, fix $N^{\prime}$ in $A$ such that $N$ and $N^{\prime}$ 
are isomorphic and $M \in N^{\prime}$. Then $M \cap \mu \in N^{\prime}$. By Lemma 3.1, $\sigma_{N^{\prime}, N}(M \cap \mu)=$ $\sigma_{N^{\prime}, N}[M \cap \mu]=M \cap \mu$. Hence $M \cap \mu$ is in $N$.

Let $G$ be a generic filter for $\mathbb{P}$. Let $J:=\left\{N: \exists\left(x_{0}, \ldots, x_{k}, A\right) \in G(N \in A)\right\}$. Then for all $M$ and $N$ in $J$, if $M \cap \omega_{1}=N \cap \omega_{1}$ then $M$ and $N$ are isomorphic. For in that case the conditions in $G$ witnessing and $M$ and $N$ are in $J$ have a common extension in $G$, and the set of models in this condition is coherent adequate. By Lemma 3.1, $M \cap \mu=N \cap \mu$. Similarly, if $M$ and $N$ are in $J$ and $M \cap \omega_{1}<N \cap \omega_{1}$, then we can find a condition $\left(x_{0}, \ldots, x_{k}, A\right)$ in $G$ such that $M$ and $N$ are in $A$. By the previous paragraph, $M \cap \mu \in N$. In particular, $M \cap \mu \subseteq N \cap \mu$.

It follows that $K:=\{N \cap \mu: N \in J\}$ is well ordered by the subset relation in order type $\omega_{1}$. Using property (IV) in the definition of a coherent adequate type forcing, an easy density argument shows that for any $i<\mu$, there is $N$ in $J$ such that $i \in N \cap \mu$. It follows that $\bigcup K=\mu$. Hence in $V[G], \mu$ is the union of $\omega_{1}$ many countable sets and therefore has size $\omega_{1}$.

Lemma 3.3. Assume that $\omega_{2} \leq 2^{\omega}<\lambda$. Suppose that $R_{0}, \ldots, R_{k}$ are subsets of $H(\lambda)$. Then for any set $z \in H(\lambda)$, there are $M$ and $N$ in $\mathcal{Y}$ satisfying the following:

(1) $z \in M \cap N$;

(2) $\{M, N\}$ is $(S)$ coherent adequate;

(3) the structures $\left(M, \in, Y \cap M, R_{0} \cap M, \ldots, R_{k} \cap M\right)$ and $\left(N, \in, Y \cap N, R_{0} \cap\right.$ $\left.N, \ldots, R_{k} \cap N\right)$ are elementary in $\left(H(\lambda), \in, Y, R_{0}, \ldots, R_{k}\right)$ and are isomorphic;

(4) there exists $\alpha \in M \cap\left(2^{\omega}\right)^{+}$and $\beta \in N \cap\left(2^{\omega}\right)^{+}$such that $\alpha \neq \beta$ and $\sigma_{M, N}(\alpha)=\beta$.

Proof. For each $i<\left(2^{\omega}\right)^{+}$fix $N_{i}$ in $\mathcal{Y}$ such that $z$ and $i$ are in $N_{i}$ and $N_{i}$ is an elementary substructure of $\left(H(\lambda), \in, Y, R_{0}, \ldots, R_{k}\right)$. This is possible since $\mathcal{Y}$ is stationary. Let $\mathfrak{N}_{i}$ denote the structure $\left(N_{i}, \in, Y \cap N_{i}, R_{0} \cap N_{i}, \ldots, R_{k} \cap N_{i}\right)$ and let $\overline{\mathfrak{N}}_{i}$ denote its transitive collapse. Since $H\left(\omega_{1}\right)$ has size $2^{\omega}$, we can fix a cofinal set $P \subseteq\left(2^{\omega}\right)^{+}$such that for all $i<j$ in $P, \overline{\mathfrak{N}}_{i}=\overline{\mathfrak{N}}_{j}$. It follows that $\mathfrak{N}_{i}$ and $\mathfrak{N}_{j}$ are isomorphic.

By the $\Delta$-system lemma, there is a cofinal set $P^{\prime} \subseteq P$ and a countable set $z$ such that for all $i<j$ in $P^{\prime}, N_{i} \cap N_{j}=z$. As there are $2^{\omega}$ many possibilities for $\sigma_{N_{i}}\left\lceil z\right.$ for $i \in P^{\prime}$, where $\sigma_{N_{i}}$ is the transitive collapsing map of $N_{i}$, we can find a cofinal set $P^{\prime \prime} \subseteq P^{\prime}$ such that for all $i<j$ in $P^{\prime \prime}, \sigma_{N_{i}}\left\lceil z=\sigma_{N_{j}}\lceil z\right.$. It follows that if $a \in N_{i} \cap N_{j}=z$, then $\sigma_{N_{i}, N_{j}}(a)=\sigma_{N_{j}}^{-1}\left(\sigma_{N_{i}}(a)\right)=a$. So $N_{i}$ and $N_{j}$ are strongly isomorphic.

Let $M$ be equal to $N_{i}$ for some fixed $i \in P^{\prime \prime}$. Then $\sup \left(M \cap\left(2^{\omega}\right)^{+}\right)<\left(2^{\omega}\right)^{+}$ since $M$ is countable. So we can find $i<j$ in $P^{\prime \prime}$ such that $\sup \left(M \cap\left(2^{\omega}\right)^{+}\right)<j$. Let $N:=N_{j}$. We will prove that $M$ and $N$ are as desired.

Properties (1) and (3) are immediate. Since $\omega_{2} \leq 2^{\omega}$ and $M$ and $N$ are isomorphic, $M \cap \omega_{2}=N \cap \omega_{2}$ by Lemma 3.1. So trivially $\{M, N\}$ is adequate. Also $R_{M}(N)$ and $R_{N}(M)$ are empty, so $\{M, N\}$ is $(S)$ adequate. As $M$ and $N$ are strongly isomorphic, $\{M, N\}$ is $(S)$ coherent adequate. This verifies property (2). For (4), let $\beta:=j$. Then $\sup \left(M \cap\left(2^{\omega}\right)^{+}\right)<\beta$. Since $\left(2^{\omega}\right)^{+}$is either equal to $\lambda$ or definable in $H(\lambda), \alpha:=\sigma_{N, M}(\beta)$ is in $M \cap\left(2^{\omega}\right)^{+}$and hence is below $\beta$. So $\alpha \neq \beta$ and $\sigma_{M, N}(\alpha)=\beta$. 
Proposition 3.4. Let $\mathbb{P}$ be an $(S, \mathcal{Y})$ coherent adequate type forcing. Let $\mu:=$ $\left(2^{\omega}\right)^{+}$. If $p$ is a condition which forces that $\left\langle f_{i}: i<\mu\right\rangle$ is a sequence of functions from $\omega$ to $\omega$, then there is $q \leq p$ and $i<j$ such that $q$ forces that $\dot{f}_{i}=\dot{f}_{j}$.

The proof is nearly identical to the proof of Proposition 2.3, replacing $\omega_{2}^{V}$ with $\mu$ and replacing references to Lemma 2.2 with references to Lemma 3.3.

Corollary 3.5. Let $\mathbb{P}$ be an $(S, \mathcal{Y})$ coherent adequate type forcing. Then $\mathbb{P}$ collapses $\left(2^{\omega}\right)^{V}$ to have size $\omega_{1}$, forces that the successor of $\left(2^{\omega}\right)^{V}$ in $V$ is equal to $\omega_{2}$, and forces $\mathrm{CH}$.

The proof of the corollary follows from Proposition 3.4 in the same way that Corollary 2.4 follows from Proposition 2.3.

\section{Some Lemmas on Closure}

The next two sections will develop the technology needed to amalgamate coherent adequate style forcings over countable elementary substructures. There are several ways in which this development goes beyond the analogous results in [6]. Besides the more general context of $H(\lambda)$, we also give an analysis of the remainder points produced under such amalgamation, and work out general results on closure. The present section handles the topic of closure 1

Let $A$ be a subset of $\mathcal{X}$ and let $x$ be a finite subset of $H(\lambda)$. We say that the pair $(x, A)$ is closed if whenever $N$ and $N^{\prime}$ are isomorphic sets in $A$ and $a \in x \cap N$, then $\sigma_{N, N^{\prime}}(a) \in x$.

Lemma 4.1. Let $A$ be a finite coherent adequate set and $x$ a finite subset of $H(\lambda)$. Let $y$ be the set

$$
x \cup\left\{\sigma_{M, M^{\prime}}(a): M, M^{\prime} \in A, M \cong M^{\prime}, a \in x \cap M\right\} .
$$

Then $(y, A)$ is closed.

Proof. Let $N$ and $N^{\prime}$ be isomorphic sets in $A$ and $a \in y \cap N$. We will prove that $\sigma_{N, N^{\prime}}(a) \in y$. If $a \in x$, then $\sigma_{N, N^{\prime}}(a) \in y$ by the definition of $y$. Otherwise there are $M$ and $M^{\prime}$ in $A$ which are isomorphic and $b$ in $x \cap M$ such that $a=\sigma_{M, M^{\prime}}(b)$. Then $a$ is in $M^{\prime} \cap N$.

Case 1: $M^{\prime}<N$. Fix $N^{*} \in A$ isomorphic to $N$ with $M^{\prime} \in N^{*}$. Then $a \in N^{*} \cap N$, so $\sigma_{N^{*}, N^{\prime}}(a)=\sigma_{N, N^{\prime}}(a)$. Let $M_{1}=\sigma_{N^{*}, N^{\prime}}\left(M^{\prime}\right)$. Then $M_{1} \in A$ and $\sigma_{M^{\prime}, M_{1}}=$ $\sigma_{N^{*}, N^{\prime}}\left\lceil M^{\prime}\right.$ by Lemma 1.10. Now $\sigma_{M, M_{1}}(b)$ is in $y$ by the definition of $y$. But $\sigma_{M, M_{1}}(b)=\sigma_{M^{\prime}, M_{1}}\left(\sigma_{M, M^{\prime}}(b)\right)=\sigma_{N^{*}, N^{\prime}}(a)=\sigma_{N, N^{\prime}}(a)$. Hence $\sigma_{N, N^{\prime}}(a) \in y$.

Case 2: $M^{\prime} \cong N$. Since $a \in M^{\prime} \cap N, \sigma_{M^{\prime}, N}(a)=a$. By the definition of $y, \sigma_{M, N^{\prime}}(b) \in y$. But $\sigma_{M, N^{\prime}}(b)=\sigma_{N, N^{\prime}}\left(\sigma_{M^{\prime}, N}\left(\sigma_{M, M^{\prime}}(b)\right)\right)=\sigma_{N, N^{\prime}}\left(\sigma_{M^{\prime}, N}(a)\right)=$ $\sigma_{N, N^{\prime}}(a)$. So $\sigma_{N, N^{\prime}}(a) \in y$.

Case 3: $N<M^{\prime}$. Fix $M_{0} \in A$ isomorphic to $M^{\prime}$ such that $N \in M_{0}$. Let $N^{*}:=\sigma_{M_{0}, M}(N)$, which is in $A$. Then $\sigma_{N^{*}, N}=\sigma_{M, M_{0}} \uparrow N^{*}$ by Lemma 1.10. Since $a \in M^{\prime} \cap N, a \in M^{\prime} \cap M_{0}$. By Lemma 1.13, $\sigma_{M_{0}, M}(a)=\sigma_{M^{\prime}, M}(a)=b$. In particular, since $a \in N, b=\sigma_{M_{0}, M}(a) \in \sigma_{M_{0}, M}(N)=N^{*}$. By the definition of $y$,

\footnotetext{
${ }^{1}$ Lemmas 4.1 and 4.2 are also true by the same arguments when coherent adequate sets are replaced by symmetric systems in the sense of [2].
} 
$\sigma_{N^{*}, N^{\prime}}(b) \in y$. But $\sigma_{N^{*}, N^{\prime}}(b)=\sigma_{N, N^{\prime}}\left(\sigma_{N^{*}, N}(b)\right)=\sigma_{N, N^{\prime}}\left(\sigma_{M, M_{0}}(b)\right)=\sigma_{N, N^{\prime}}(a)$. So $\sigma_{N, N^{\prime}}(a) \in y$.

The next lemma analyzes closure in the context of amalgamation over countable models.

Lemma 4.2. Let $A$ be a coherent adequate set, $N \in A$, and suppose that $B$ is a coherent adequate set with $A \cap N \subseteq B \subseteq N$. Let $x$ and $y$ be subsets of $H(\lambda)$ such that $(x, A)$ is closed, $(y, B)$ is closed, and $x \cap N \subseteq y \subseteq N$. Define $C$ by

$$
\{M \in A: N \leq M\} \cup\left\{\sigma_{N, N^{\prime}}(K): N^{\prime} \in A, N \cong N^{\prime}, K \in B\right\} .
$$

Define $z$ by

$$
x \cup\left\{\sigma_{N, N^{\prime}}(a): N^{\prime} \in A, N \cong N^{\prime}, a \in y\right\} .
$$

Then the pair $(z, C)$ is closed, $x \cup y \subseteq z$, and $z \cap N=y$.

Proof. Assume that $K$ and $M$ are in $C$ and are isomorphic, and $a$ is in $z \cap K$. We will show that $\sigma_{K, M}(a) \in z$.

Case 1: $N \leq K$. Then $N \leq M$. So both $K$ and $M$ are in $A$. If $a \in x$, then we are done since $(x, A)$ is closed. So assume that $a=\sigma_{N, N^{\prime}}\left(a_{0}\right)$ for some $N^{\prime}$ in $A$ isomorphic to $N$ and $a_{0} \in y$.

Subcase 1a: $K$ and $M$ are isomorphic to $N$. Since $a \in K \cap N^{\prime}, \sigma_{N^{\prime}, K}(a)=$ $a$ by Lemma 1.13. By the definition of $z, \sigma_{N, M}\left(a_{0}\right) \in z$. But $\sigma_{N, M}\left(a_{0}\right)=$ $\sigma_{K, M}\left(\sigma_{N^{\prime}, K}\left(\left(\sigma_{N, N^{\prime}}\left(a_{0}\right)\right)\right)=\sigma_{K, M}\left(\sigma_{N^{\prime}, K}(a)\right)=\sigma_{K, M}(a)\right.$. So $\sigma_{K, M}(a) \in z$.

Subcase 1b: $N<K$. Since $A$ is adequate, fix $J \in A$ isomorphic to $K$ such that $N^{\prime} \in J$. Let $N^{\prime \prime}:=\sigma_{J, K}\left(N^{\prime}\right)$ and $N^{\prime \prime \prime}:=\sigma_{K, M}\left(N^{\prime \prime}\right)$. Then $N^{\prime \prime}$ and $N^{\prime \prime \prime}$ are in $A$ and are isomorphic to $N$. Moreover $\sigma_{N^{\prime}, N^{\prime \prime}}=\sigma_{J, K} \uparrow N^{\prime}$ and $\sigma_{N^{\prime \prime}, N^{\prime \prime \prime}}=\sigma_{K, M} \uparrow$ $N^{\prime \prime}$. By definition of $z, a_{1}:=\sigma_{N, N^{\prime \prime \prime}}\left(a_{0}\right)$ is in $z$. Since $a$ is in $N^{\prime} \cap K, a$ is in $J \cap K$, so $\sigma_{J, K}(a)=a$. Hence $a_{1}=\sigma_{N, N^{\prime \prime \prime}}\left(a_{0}\right)=\sigma_{N^{\prime \prime}, N^{\prime \prime \prime}}\left(\sigma_{N^{\prime}, N^{\prime \prime}}\left(\sigma_{N, N^{\prime}}\left(a_{0}\right)\right)\right)=$ $\sigma_{N^{\prime \prime}, N^{\prime \prime \prime}}\left(\sigma_{N^{\prime}, N^{\prime \prime}}(a)\right)=\sigma_{K, M}\left(\sigma_{J, K}(a)\right)=\sigma_{K, M}(a)$. So $\sigma_{K, M}(a) \in z$.

Case 2: $K=\sigma_{N, N^{*}}(L)$ for some $N^{*} \in A$ isomorphic to $N$ and $L \in B$. Since $K$ and $M$ are isomorphic, $M \cap \omega_{1}=K \cap \omega_{1}<N \cap \omega_{1}$. So also $M=\sigma_{N, N^{\prime}}(P)$ for some $N^{\prime} \in A$ isomorphic to $N$ and $P \in B$. Then $L \cap \omega_{1}=P \cap \omega_{1}$. Since $B$ is coherent adequate, $L$ and $P$ are isomorphic.

Subcase 2a: $a=\sigma_{N, N^{\prime \prime}}\left(a_{0}\right)$ for some $N^{\prime \prime} \in A$ isomorphic to $N$ and $a_{0} \in y$. Since $a \in K=\sigma_{N, N^{*}}(L), a \in N^{\prime \prime} \cap N^{*}$. By Lemma 1.13, $\sigma_{N^{*}, N}(a)=\sigma_{N^{\prime \prime}, N}(a)=a_{0}$. So $\sigma_{K, L}(a)=\sigma_{N^{*}, N}(a)=a_{0}$. It follows that $\sigma_{K, M}(a)=\sigma_{P, M}\left(\sigma_{L, P}\left(\sigma_{K, L}(a)\right)\right)=$ $\sigma_{P, M}\left(\sigma_{L, P}\left(a_{0}\right)\right)=\sigma_{N, N^{\prime}}\left(\sigma_{L, P}\left(a_{0}\right)\right)$. Since $(y, B)$ is closed, $\sigma_{L, P}\left(a_{0}\right)$ is in $y$. So by the definition of $z, \sigma_{N, N^{\prime}}\left(\sigma_{L, P}\left(a_{0}\right)\right)$ is in $z$. That is, $\sigma_{K, M}(a)$ is in $z$.

Subcase 2b: $a$ is in $x$. Since $a \in K=\sigma_{N, N^{*}}(L)$, it follows that $a \in N^{*}$. As $(x, A)$ is closed, $a_{0}:=\sigma_{N^{*}, N}(a)$ is in $x \cap N$ and hence in $y$. But now $a=\sigma_{N, N^{*}}\left(a_{0}\right), N^{*}$ is in $A, N^{*}$ is isomorphic to $N$, and $a_{0} \in y$. So we are done by subcase $2 \mathrm{a}$.

Now let us prove that $x \cup y \subseteq z$ and $z \cap N=y$. The set $x$ is a subset of $z$ by definition. And if $a \in y$, then $a=\sigma_{N, N}(a)$ is in $z$. So $x \cup y \subseteq z$. The set $y$ is a subset of $N$ by definition, and we just showed that $y$ is a subset of $z$. So $y \subseteq z \cap N$. On the other hand, suppose that $a \in z \cap N$. If $a \in x$, then $a \in x \cap N$ and hence 
$a \in y$. Otherwise $a=\sigma_{N, N^{\prime}}\left(a_{0}\right)$, where $N^{\prime} \in A$ is isomorphic to $N$ and $a_{0} \in y$. But then $a \in N^{\prime} \cap N$, so $\sigma_{N^{\prime}, N}(a)=a$. Hence $a=a_{0}$, so $a \in y$.

\section{Amalgamating over Countable Models}

In this section we prove an amalgamation result for countable models. The difference between this proposition and the analogous result from [6] is that here we provide an analysis of remainder points.

The next lemma considers a special case of the general result.

Lemma 5.1. Let $N, N^{\prime}, N^{*}$ be isomorphic in $\mathcal{X}$ such that $\left\{N, N^{\prime}, N^{*}\right\}$ is coherent adequate. Assume that $K$ and $L$ are in $N \cap \mathcal{X}$ and $\{K, L\}$ is adequate. Let $M:=$ $\sigma_{N, N^{\prime}}(K)$ and $P:=\sigma_{N, N^{*}}(L)$. Then $\{M, P\}$ is adequate. Moreover, if $\zeta$ is in $R_{P}(M) \cap \beta_{N^{\prime}, N^{*}}$, then $\sigma_{N^{\prime}, N}(\zeta)$ is in $R_{L}(K)$.

Proof. Let $\sigma:=\sigma_{N^{\prime}, N}\left\lceil N^{\prime} \cap N^{*}\right.$. By Lemma 1.13, $\sigma$ is equal to $\sigma_{N^{*}, N}\left\lceil N^{\prime} \cap N^{*}\right.$.

Note that $\beta_{M, P} \leq \beta_{N^{\prime}, N^{*}}$, since $M \in N^{\prime}$ and $P \in N^{*}$. Therefore $M \cap \beta_{M, P}$ is a countable subset of $\beta_{N^{\prime}, N^{*}}$. So $M \cap \beta_{M, P}$ is in $N^{\prime} \cap P_{\omega_{1}}\left(\beta_{N^{\prime}, N^{*}}\right)$. As $N^{\prime}$ and $N^{*}$ are strongly isomorphic, $M \cap \beta_{M, N} \in N^{*}$ by Lemma 1.18. For the same reason, $P \cap \beta_{M, P}$ is in $N^{\prime}$. Since $\beta_{M, P}$ is definable from $M \cap \beta_{M, P}$ as $\min \left(\Lambda \backslash \sup \left(M \cap \beta_{M, P}\right)\right)$, we have that $\beta_{M, P} \in N^{\prime} \cap N^{*}$. Also $\sigma\left(M \cap \beta_{M, P}\right)=\sigma_{N^{\prime}, N}(M) \cap \sigma\left(\beta_{M, P}\right)=K \cap \sigma\left(\beta_{M, P}\right)$, and similarly $\sigma\left(P \cap \beta_{M, P}\right)=L \cap \sigma\left(\beta_{M, P}\right)$.

We claim that $\sigma\left(\beta_{M, P}\right) \leq \beta_{K, L}$. Since $\beta_{M, P} \in \Lambda_{M}, \sigma\left(\beta_{M, P}\right) \in \sigma_{N^{\prime}, N}\left(\Lambda_{M}\right)=$ $\Lambda_{K}$. Similarly, $\sigma\left(\beta_{M, P}\right) \in \Lambda_{L}$. Since $\beta_{K, L}=\max \left(\Lambda_{K} \cap \Lambda_{L}\right), \sigma\left(\beta_{M, P}\right) \leq \beta_{K, L}$.

Let us show that $\{M, P\}$ is adequate. We will use the fact that $\{K, L\}$ is adequate and consider three cases.

Case 1: $K<L$. Then $K \cap \beta_{K, L} \in L$. Since $\sigma\left(\beta_{M, P}\right) \leq \beta_{K, L}, K \cap \sigma\left(\beta_{M, P}\right) \in L$. But $\sigma\left(M \cap \beta_{M, P}\right)=K \cap \sigma\left(\beta_{M, P}\right)$ and $\sigma=\sigma_{N^{*}, N}\left\lceil N^{\prime} \cap N^{*}\right.$. Applying $\sigma_{N, N^{*}}$, we get that $\sigma^{-1}\left(K \cap \sigma\left(\beta_{M, N}\right)\right) \in \sigma_{N, N^{*}}(L)$, that is $M \cap \beta_{M, P} \in P$.

Case 2: $L<K$. This case follows by a symmetric argument.

Case 3: $K \sim L$. As $\sigma\left(\beta_{M, P}\right) \leq \beta_{K, L}$, it follows that $K \cap \sigma\left(\beta_{M, P}\right)=L \cap \sigma\left(\beta_{M, P}\right)$. As noted above, $\sigma\left(M \cap \beta_{M, P}\right)=K \cap \sigma\left(\beta_{M, P}\right)$ and $\sigma\left(P \cap \beta_{M, P}\right)=L \cap \sigma\left(\beta_{M, P}\right)$. So applying $\sigma^{-1}$, we get that $M \cap \beta_{M, P}=P \cap \beta_{M, P}$.

This completes the proof that $\{M, P\}$ is adequate. Now let $\zeta$ be in $R_{P}(M) \cap$ $\beta_{N^{\prime}, N^{*}}$. We will show that $\zeta_{0}:=\sigma(\zeta)$ is in $R_{L}(K)$. Since $\zeta \in M$, applying $\sigma_{N^{\prime}, N}$, it follows that $\zeta_{0} \in K$. Note that since $\beta_{M, P} \leq \zeta, \sigma\left(\beta_{M, P}\right) \leq \zeta_{0}$. So $\zeta_{0} \in K \backslash \sigma\left(\beta_{M, P}\right)$.

Case $A$ : $M \leq P$. Then $K \leq L$. We claim that in this case, $\sigma\left(\beta_{M, P}\right)=\beta_{K, L}$. We already know that $\sigma\left(\beta_{M, P}\right) \leq \beta_{K, L}$. Suppose for a contradiction that $\sigma\left(\beta_{M, P}\right)<$ $\beta_{K, L}$. By Lemma 1.2, $K \cap\left[\sigma\left(\beta_{M, P}\right), \beta_{K, L}\right)$ is nonempty. So letting $\tau_{0}=\min (K \backslash$ $\left.\sigma\left(\beta_{M, P}\right)\right), \tau_{0}<\beta_{K, L}$. Since $\sigma(\zeta)=\zeta_{0} \in K \backslash \sigma\left(\beta_{M, P}\right), \tau_{0} \leq \zeta_{0}$ by the minimality of $\tau_{0}$. So $\tau:=\sigma_{N, N^{\prime}}\left(\tau_{0}\right) \leq \zeta$. Also since $\tau_{0} \in K, \tau \in M$. And because $\sigma\left(\beta_{M, P}\right) \leq \tau_{0}$, $\beta_{M, P} \leq \tau$. So $\tau$ is in $M \backslash \beta_{M, P}$.

Since $K \leq L, \tau_{0} \in K \cap \beta_{K, L}$ implies that $\tau_{0} \in L$. So $\tau_{0} \in K \cap L$. As $\tau \leq \zeta$ and $\zeta<\beta_{N^{\prime}, N^{*}}, \tau<\beta_{N^{\prime}, N^{*}}$. So $\tau=\sigma_{N, N^{\prime}}\left(\tau_{0}\right)=\sigma_{N, N^{*}}\left(\tau_{0}\right)$ by Lemma 1.13. Since 
$\tau_{0} \in L, \tau=\sigma_{N, N^{*}}\left(\tau_{0}\right) \in P$. So $\tau \in\left(M \cap P \cap \omega_{2}\right) \backslash \beta_{M, P}$, which is impossible. This contradiction shows that $\sigma\left(\beta_{M, P}\right)=\beta_{K, L}$.

Now we show that $\zeta_{0} \in R_{L}(K)$. If $\zeta=\min \left(M \backslash \beta_{M, P}\right)$, then applying $\sigma_{N^{\prime}, N}$ we get that $\zeta_{0}=\min \left(K \backslash \sigma\left(\beta_{M, P}\right)\right)=\min \left(K \backslash \beta_{K, L}\right)$. So $\zeta_{0} \in R_{L}(K)$. Now assume that there is $\gamma \in P \backslash \beta_{M, P}$ such that $\zeta=\min (M \backslash \gamma)$. Since $\zeta<\beta_{N^{\prime}, N^{*}}, \gamma<\beta_{N^{\prime}, N^{*}}$. Let $\gamma_{0}:=\sigma(\gamma)$. Applying $\sigma_{N^{\prime}, N}, \zeta_{0}=\min \left(K \backslash \gamma_{0}\right)$. As $\sigma\left(\beta_{M, P}\right)=\beta_{K, L}$ and $\beta_{M, P} \leq \gamma, \beta_{K, L} \leq \gamma_{0}$. Also $\gamma \in P$ implies that $\gamma_{0}=\sigma_{N^{*}, N}(\gamma) \in \sigma_{N^{*}, N}(P)=L$. So $\gamma_{0} \in L \backslash \beta_{K, L}$. Therefore $\zeta_{0} \in R_{L}(K)$.

Case 2: $P<M$. Then $L<K$. As $\zeta \in R_{P}(M)$, there is $\gamma \in P \backslash \beta_{M, P}$ such that $\zeta=\min (M \backslash \gamma)$. Since $\gamma<\zeta<\beta_{N^{\prime}, N^{*}}, \gamma \in P \cap \beta_{N^{\prime}, N^{*}}$. Applying $\sigma_{N^{*}, N}$, $\gamma_{0}:=\sigma(\gamma)$ is in $\sigma_{N^{*}, N}(P)=L$. Since $\gamma \in P \backslash \beta_{M, P}, \gamma$ is not in $M$. Applying $\sigma_{N^{\prime}, N}, \gamma_{0}$ is not in $\sigma_{N^{\prime}, N}(M)=K$. So $\gamma_{0} \in L \backslash K$. Since $L<K$, this implies that $\beta_{K, L} \leq \gamma_{0}$. Therefore $\gamma_{0} \in L \backslash \beta_{K, L}$. Since $\zeta=\min (M \backslash \gamma), \zeta_{0}=\min \left(K \backslash \gamma_{0}\right)$. Hence $\zeta_{0} \in R_{L}(K)$.

Proposition 5.2. Let $A$ be a coherent adequate set and $N \in A$. Suppose that $B$ is a coherent adequate set and $A \cap N \subseteq B \subseteq N$. Let $C$ be the set

$$
\{M \in A: N \leq M\} \cup\left\{\sigma_{N, N^{\prime}}(K): N^{\prime} \in A, N \cong N^{\prime}, K \in B\right\} .
$$

Then $C$ is a coherent adequate set, $A \cup B \subseteq C$, and $C \cap N=B$. Moreover, $R_{C}$ is a subset of

$$
R_{A} \cup\left\{\min (K \backslash \zeta): K \in C, \zeta \in R_{A}\right\} \cup\left\{\sigma_{N, N^{\prime}}(\tau): N^{\prime} \in A, N \cong N^{\prime}, \tau \in R_{B}\right\} .
$$

Proof. Let $M$ and $P$ be in $C$ such that $M \cap \omega_{1} \leq P \cap \omega_{1}$. We will show that $\{M, P\}$ is adequate and that the remainder points of $M$ and $P$ are as required.

Case 1: $N \cap \omega_{1} \leq M \cap \omega_{1}$. Then $N \cap \omega_{1} \leq M \cap \omega_{1} \leq P \cap \omega_{1}$. Hence $M$ and $P$ are both in $A$. So obviously $\{M, P\}$ is adequate and $R_{M}(P) \cup R_{P}(M) \subseteq R_{A}$.

Case 2: $M \cap \omega_{1}<N \cap \omega_{1} \leq P \cap \omega_{1}$. Then clearly $P \in A, N \leq P$, and $M=\sigma_{N, N^{\prime}}(K)$ for some $N^{\prime} \in A$ isomorphic to $N$ and $K \in B$. Since $N \leq P$ and $N$ and $N^{\prime}$ are isomorphic, $N^{\prime} \leq P$. And as $M \in N^{\prime}, \beta_{M, P} \leq \beta_{N^{\prime}, P}$. So $M \cap \beta_{M, P}$ is in $N^{\prime} \cap P_{\omega_{1}}\left(\beta_{N^{\prime}, P}\right)$. As $N^{\prime} \leq P, M \cap \beta_{M, P} \in P$ by Lemma 1.19. So $M<P$.

Let $\zeta \in R_{M}(P)$ be given, and we will show that $\zeta \in R_{A}$. Since $M<P$, there is $\gamma \in M \backslash \beta_{M, P}$ such that $\zeta=\min (P \backslash \gamma)$. As $\beta_{M, P} \leq \gamma, \gamma$ is not in $P$. Since $N^{\prime} \leq P$ and $\gamma \in N^{\prime} \backslash P, \beta_{N^{\prime}, P} \leq \gamma$. So $\gamma \in N^{\prime} \backslash \beta_{N^{\prime}, P}$. Therefore $\zeta$ is in $R_{N^{\prime}}(P)$ and hence in $R_{A}$.

Now let $\zeta \in R_{P}(M)$ be given. Since $M<P$, there is $\gamma \in P \backslash \beta_{M, P}$ such that $\zeta=\min (M \backslash \gamma)$. As $\zeta$ is in $N^{\prime} \backslash P$ and $N^{\prime} \leq P, \beta_{N^{\prime}, P} \leq \zeta$. If $\gamma<\beta_{N^{\prime}, P}$, then $\zeta=\min \left(M \backslash \beta_{N^{\prime}, P}\right)$. Let $\tau:=\min \left(N^{\prime} \backslash \beta_{N^{\prime}, P}\right)$. Then $\tau \in R_{A}$. Since $M \subseteq N^{\prime}$, $\zeta=\min (M \backslash \tau)$. Thus $\zeta$ is as required. Now assume that $\beta_{N^{\prime}, P} \leq \gamma$. Then $\gamma$ is in $P \backslash \beta_{N^{\prime}, P}$. Hence $\pi:=\min \left(N^{\prime} \backslash \gamma\right)$ is in $R_{A}$. But then $\zeta=\min (M \backslash \pi)$, so $\zeta$ is as required.

Case 3: $M \cap \omega_{1} \leq P \cap \omega_{1}<N \cap \omega_{1}$. Then $M=\sigma_{N, N^{\prime}}(K)$ and $P=\sigma_{N, N^{*}}(L)$ for some $N^{\prime}$ and $N^{*}$ in $A$ isomorphic to $N$ and $K$ and $L$ in $B$. By Lemma 5.1, $\{M, P\}$ is adequate. Moreover, if $\zeta$ is in $R_{P}(M) \cap \beta_{N^{\prime}, N^{*}}$ then $\sigma_{N^{\prime}, N}(\zeta) \in R_{B}$, and if $\zeta$ is in $R_{M}(P) \cap \beta_{N^{\prime}, N^{*}}$ then $\sigma_{N^{*}, N}(\zeta) \in R_{B}$. In either case, $\zeta$ is as required.

Let $\zeta \in R_{P}(M) \backslash \beta_{N^{\prime}, N^{*}}$ be given. Since $M \in N^{\prime}$ and $P \in N^{*}, \beta_{M, P} \leq \beta_{N^{\prime}, N^{*}}$. If $\zeta=\min \left(M \backslash \beta_{M, P}\right)$ or if $\zeta=\min (M \backslash \gamma)$ for some $\gamma \in P \backslash \beta_{M, P}$ which is below 
$\beta_{N^{\prime}, N^{*}}$, then $\zeta=\min \left(M \backslash \beta_{N^{\prime}, N^{*}}\right)$. So letting $\tau:=\min \left(N^{\prime} \backslash \beta_{N^{\prime}, N^{*}}\right), \tau \in R_{A}$ and clearly $\zeta=\min (M \backslash \tau)$. So $\zeta$ is as required. Otherwise there is $\gamma \in P \backslash \beta_{M, P}$ such that $\beta_{N^{\prime}, N^{*}} \leq \gamma$ and $\zeta=\min (M \backslash \gamma)$. Since $\gamma \in P$ and $P \in N^{*}, \gamma \in N^{*} \backslash \beta_{N^{\prime}, N^{*}}$. Let $\pi:=\min \left(N^{\prime} \backslash \gamma\right)$. Then $\pi \in R_{A}$ and clearly $\zeta=\min (M \backslash \pi)$, and we are done. The proof that the remainder points in $R_{M}(P)$ are as required follows by a symmetric argument, since we never used in this paragraph the assumption that $M \cap \omega_{1} \leq P \cap \omega_{1}$.

Now we show that $A \cup B \subseteq C$. If $K \in B$, then $K=\sigma_{N, N}(K)$ is in $C$. Let $M \in A$. If $N \cap \omega_{1} \leq M \cap \omega_{1}$, then $M \in C$. Otherwise $M \cap \omega_{1}<N \cap \omega_{1}$. Since $A$ is coherent, there exists $N^{\prime} \in A$ isomorphic to $N$ such that $M \in N^{\prime}$. Let $K:=\sigma_{N^{\prime}, N}(M)$. Then $K$ is in $A \cap N$ and hence in $B$. So $\sigma_{N, N^{\prime}}(K)=M$ is in $C$.

It remains to prove that $C$ is coherent and $C \cap N=B$. We apply Lemma 4.2 in the case where $x=A$ and $y=B$. Then clearly the set $z$ defined there is equal to $C$. Since $A$ and $B$ are coherent, the pairs $(A, A)$ and $(B, B)$ are closed. So by Lemma 4.2, $(C, C)$ is closed and $C \cap N=B$. Therefore if $M$ and $M^{\prime}$ are in $C$ and are isomorphic, and $K \in M \cap C$, then $\sigma_{M, M^{\prime}}(K) \in C$.

We prove the remaining properties in the definition of coherence. Suppose that $M$ and $P$ are in $C$ and $M \cap \omega_{1}=P \cap \omega_{1}$. If $N \leq M$, then $M$ and $P$ are both in $A$ and hence are strongly isomorphic. Otherwise $M<N$ and $P<N$, which implies that $M=\sigma_{N, N^{\prime}}(K)$ and $P=\sigma_{N, N^{*}}(L)$ for some $N^{\prime}$ and $N^{*}$ in $A$ isomorphic to $N$ and $K$ and $L$ in $B$. Then $K \cap \omega_{1}=M \cap \omega_{1}=P \cap \omega_{1}=L \cap \omega_{1}$. Since $B$ is coherent, $K$ and $L$ are strongly isomorphic. By Lemma 1.14, $M$ and $P$ are strongly isomorphic.

Now assume that $M$ and $P$ are in $C$ and $M<P$. We will show that there is $P^{\prime}$ in $C$ isomorphic to $P$ such that $M \in P^{\prime}$. If $N \cap \omega_{1} \leq M \cap \omega_{1}$, then $M$ and $P$ are both in $A$, and we are done since $A$ is coherent. Suppose that $M \cap \omega_{1}<N \cap \omega_{1} \leq P \cap \omega_{1}$. Then $M=\sigma_{N, N^{\prime}}(K)$ for some $N^{\prime} \in A$ isomorphic to $N$ and $K \in B$. If $N^{\prime}$ is isomorphic to $P$ then we are done. Otherwise $N^{\prime}<P$, so there is $P^{\prime} \in A$ isomorphic to $P$ with $N^{\prime} \in P^{\prime}$. Then $M \in P^{\prime}$.

Finally, assume that $M=\sigma_{N, N^{\prime}}(K)$ and $P=\sigma_{N, N^{*}}(L)$ for some $K$ and $L$ in $B$ and $N^{\prime}$ and $N^{*}$ in $A$ isomorphic to $N$. Since $M<P, K<L$. As $B$ is coherent, fix $L^{\prime}$ in $B$ isomorphic to $L$ such that $K \in L^{\prime}$. Then $M=\sigma_{N, N^{\prime}}(K) \in \sigma_{N, N^{\prime}}\left(L^{\prime}\right)$ and $\sigma_{N, N^{\prime}}\left(L^{\prime}\right) \in C$. Since $L$ and $L^{\prime}$ are strongly isomorphic in $N, \sigma_{N, N^{\prime}}\left(L^{\prime}\right)$ and $\sigma_{N, N^{*}}(L)=P$ are strongly isomorphic by Lemma 1.14 .

\section{Adding a square Sequence}

We review the forcing poset from [ $[\underline{6}$ for adding a square sequence with finite conditions, and show that it is in the class of coherent adequate type forcing posets. As a consequence, this forcing poset preserves $\mathrm{CH}$.

By a triple we mean a sequence $\langle\alpha, \gamma, \beta\rangle$, where $\alpha \in \Lambda$ and $\gamma<\beta<\alpha$. Given distinct triples $\langle\alpha, \gamma, \beta\rangle$ and $\left\langle\alpha^{\prime}, \gamma^{\prime}, \beta^{\prime}\right\rangle$, we say that the triples are nonoverlapping if either $\alpha \neq \alpha^{\prime}$, or $\alpha=\alpha^{\prime}$ and $[\gamma, \beta) \cap\left[\gamma^{\prime}, \beta^{\prime}\right)=\emptyset$; otherwise they are overlapping.

Definition 6.1. Let $\mathbb{P}$ be the forcing poset whose conditions are pairs $(x, A)$ satisfying:

(1) $x$ is a finite set of nonoverlapping triples;

(2) A is a finite coherent adequate set; 
(3) for all $M \in A$ and $\langle\alpha, \gamma, \beta\rangle \in x$ such that $\alpha \in M$, either $\gamma$ and $\beta$ are in $M$ or $\sup (M \cap \alpha)<\gamma$

(4) if $M$ and $M^{\prime}$ are isomorphic sets in $A$, then for any triple $\langle\alpha, \gamma, \beta\rangle \in M \cap x$, $\sigma_{M, M^{\prime}}(\langle\alpha, \gamma, \beta\rangle) \in x$.

Let $(y, B) \leq(x, A)$ if $x \subseteq y$ and $A \subseteq B$.

We proved in [6] that $\mathbb{P}$ is strongly proper, $\omega_{2}$-c.c., and forces $\square_{\omega_{1}}$.

Proposition 6.2. The forcing poset $\mathbb{P}$ is a coherent adequate type forcing poset.

Proof. Let $(x, A)$ be condition in $\mathbb{P}$ and we will verify requirements $(\mathrm{I})-(\mathrm{V})$ in the definition of a coherent adequate type forcing. The proof of Proposition 4.3 in [6 shows that if $(x, A)$ is a condition and $M \in A$, then $(x, A)$ is strongly $M$-generic. So requirement (V) holds. Obviously $x$ is a subset of $H\left(\omega_{2}\right)$, and by assumption $A$ is a coherent adequate set. Thus requirements (I) and (II) are immediate.

(III) Suppose that $(y, B) \leq(x, A), N$ and $N^{\prime}$ are isomorphic sets in $B$, and $(x, A) \in N$. Properties (1)-(4) in the definition of $\mathbb{P}$ are all first order definable in $H\left(\omega_{2}\right)$, except the part of (2) which asserts membership in $\mathcal{X}$. Since $\sigma_{N, N^{\prime}}$ is an isomorphism which preserves membership in $\mathcal{X}$ and $N$ and $N^{\prime}$ are elementary substructures of $H\left(\omega_{2}\right), \sigma_{M, N}((x, A))$ is in $\mathbb{P}$. Also $\sigma_{M, N}((x, A))=\left(\sigma_{M, N}[x], \sigma_{M, N}[A]\right)$. Since $(y, B)$ is closed and $B$ is coherent, $x \subseteq y$ and $A \subseteq B$ imply that $\sigma_{M, N}[x] \subseteq y$ and $\sigma_{M, N}[A] \subseteq B$. Consequently, $(y, B) \leq \sigma_{M, N}((x, a))$.

(IV) Suppose that $M_{0}, \ldots, M_{n}$ are isomorphic sets in $\mathcal{X}$ such that $\left\{M_{0}, \ldots, M_{n}\right\}$ is coherent adequate and $(x, A) \in M_{0} \cap \cdots \cap M_{n}$. We claim that $\left(x, A \cup\left\{M_{0}, \ldots, M_{n}\right\}\right)$ satisfies properties (1)-(4) in the definition of $\mathbb{P}$. By Lemma 1.17, $A \cup\left\{M_{0}, \ldots, M_{n}\right\}$ is coherent adequate so (2) is satisfied. (1) is immediate. For (3), any triple in $x$ is a member of $M_{0}, \ldots, M_{n}$, so there is nothing to check. And for (4), if $i, j \leq n$ and $a \in x \cap M_{i}$, then $a \in M_{i} \cap M_{j}$ so $\sigma_{M_{i}, M_{j}}(a)=a$ is in $x$.

\section{Adding a Club PRESERving CH}

A forcing poset for adding a club to a fat subset of $\omega_{2}$ using adequate sets as side conditions appears in [4]. Friedman [3] asked whether it is possible to add a club to $\omega_{2}$ with finite conditions while preserving $\mathrm{CH}$. In this section we adapt of variation of the poset from [4] to solve this problem.

Fix a stationary set $S \subseteq \omega_{2}$ which is fat. So for every club set $C \subseteq \omega_{2}, S \cap C$ contains a closed subset with order type $\omega_{1}+1$. Without loss of generality we may assume that $S \cap \operatorname{cof}\left(\omega_{1}\right)$ is stationary and for all $\alpha \in S \cap \operatorname{cof}\left(\omega_{1}\right), S \cap \alpha$ contains a closed cofinal subset of $\alpha$. For the assumption of $S$ being fat implies that there is a stationary subset of $S$ satisfying this property.

The general framework of coherent adequate sets introduced in the first section involves the parameters $\lambda$ and $Y$. For this application we let $\lambda:=\omega_{2}$ and let $Y$ code $S$ together with a well-ordering of $H\left(\omega_{2}\right)$. So $\mathcal{X}$ consists of countable elementary substructures of $H\left(\omega_{2}\right)$, and isomorphisms between members of $\mathcal{X}$ preserve membership in $S$.

Let $\mathcal{Y}$ denote the set of $M$ in $\mathcal{X}$ such that for all $\alpha \in(M \cap S) \cup\left\{\omega_{2}\right\}, \sup (M \cap$ $\alpha) \in S$. A straightforward argument using the properties of $S$ shows that $\mathcal{Y}$ is stationary in $P_{\omega_{1}}\left(H\left(\omega_{2}\right)\right)$. Also since isomorphisms between members of $\mathcal{X}$ preserve membership in $S$, it is easy to check that $\mathcal{Y}$ is closed under isomorphisms. 
For an ordinal $\alpha$ and a set $N$ such that $N \cap \omega_{2}$ is not a subset of $\alpha$, let $\alpha_{N}$ denote $\min (N \backslash \alpha)$. Given pairs of ordinals $\left\langle\alpha, \alpha^{\prime}\right\rangle$ and $\left\langle\gamma, \gamma^{\prime}\right\rangle$, we say that the pairs overlap if $\alpha<\gamma \leq \alpha^{\prime}$ or $\gamma<\alpha \leq \gamma^{\prime}$; otherwise they are nonoverlapping.

Definition 7.1. Let $\mathbb{P}$ be the forcing poset consisting of conditions of the form $p=\left(x_{p}, A_{p}\right)$ satisfying:

(1) $x_{p}$ is a finite set of nonoverlapping pairs of the form $\left\langle\alpha, \alpha^{\prime}\right\rangle$, where $\alpha \leq$ $\alpha^{\prime}<\omega_{2}$ and $\alpha \in S$

(2) $A_{p}$ is a finite coherent adequate subset of $\mathcal{Y}$;

(3) if $\left\langle\alpha, \alpha^{\prime}\right\rangle \in x_{p}, N \in A_{p}$, and $N \cap \omega_{2} \nsubseteq \alpha$, then $N \cap\left[\alpha, \alpha^{\prime}\right] \neq \emptyset$ implies that $\alpha$ and $\alpha^{\prime}$ are in $N$, and $N \cap\left[\alpha, \alpha^{\prime}\right]=\emptyset$ implies that $\left\langle\alpha_{N}, \alpha_{N}\right\rangle \in x_{p}$;

(4) for all $\zeta$ in $R_{A_{p}},\langle\zeta, \zeta\rangle \in x$;

(5) if $M$ and $N$ are in $A_{p}$ and are isomorphic, then for any $a \in M \cap x_{p}$, $\sigma_{M, N}(a) \in x_{p}$.

Let $q \leq p$ if $x_{p} \subseteq x_{q}$ and $A_{p} \subseteq A_{q}$.

Observe that if $(x, A)$ is a condition and $N \in A$, then $(x \cap N, A \cap N)$ is also a condition. Also, if $(x, A)$ is in $\mathbb{P}$ and $B$ is a subset of $A$ which is coherent adequate, then $(x, B)$ is a condition.

Note that in requirement (3), if the pair is of the form $\langle\alpha, \alpha\rangle$, then the conclusion in either case is equivalent to requiring that $\left\langle\alpha_{N}, \alpha_{N}\right\rangle \in x$.

Let $\dot{C}_{S}$ be a $\mathbb{P}$-name such that $\mathbb{P}$ forces

$$
\dot{C}_{S}=\left\{\alpha: \exists p \in \dot{G} \exists \alpha^{\prime}\left\langle\alpha, \alpha^{\prime}\right\rangle \in x_{p}\right\} .
$$

Clearly $\dot{C}_{S}$ is forced to be a subset of $S$. We will show that $\mathbb{P}$ is an $(S, \mathcal{Y})$ coherent adequate type forcing and $\mathbb{P}$ forces that $\dot{C}_{S}$ is club in $\omega_{2}$.

Proposition 7.2. Let $q=(x, A)$ be a condition and assume that $N \in A$. Then $q$ is strongly $N$-generic.

Proof. Fix a set $D$ which is dense in the poset $N \cap \mathbb{P}$, and we will show that $D$ is predense below $q$. Let $r \leq q$ be given. Then $r \mid N:=\left(x_{r} \cap N, A_{r} \cap N\right)$ is a condition in $N$. Since $D$ is dense, fix $w$ in $D$ below $r \uparrow N$. We will prove that $r$ and $w$ are compatible.

Let $C$ denote the set

$$
\left\{M \in A_{r}: N \leq M\right\} \cup\left\{\sigma_{N, N^{\prime}}(K): N^{\prime} \in A_{r}, N \cong N^{\prime}, K \in A_{w}\right\} .
$$

Let $z$ denote the set

$$
x_{r} \cup\left\{\sigma_{N, N^{\prime}}(a): N^{\prime} \in A_{r}, N \cong N^{\prime}, a \in x_{w}\right\} .
$$

Let $s:=(z, C)$. We will show that $s$ is a condition and $s \leq r, w$.

By Proposition 5.2, $C$ is a finite coherent adequate set, $A_{r} \cup A_{w} \subseteq C$, and $C \cap N=A_{w}$. Also $C$ is a subset of $\mathcal{Y}$ since $\mathcal{Y}$ is closed under isomorphisms. So $s$ satisfies requirement (2) in the definition of $\mathbb{P}$. By Lemma 4.2, the pair $(z, C)$ is closed, $x_{r} \cup x_{w} \subseteq z$, and $z \cap N=x_{w}$. Since $(z, C)$ is closed, if $M$ and $M^{\prime}$ are isomorphic sets in $C$, then for any $a \in M \cap z, \sigma_{M, M^{\prime}}(a) \in z$. Thus $s$ satisfies requirement (5) in the definition of $\mathbb{P}$. Since $x_{r} \cup x_{w} \subseteq z$ and $A_{r} \cup A_{w} \subseteq C$, it follows that if $s$ is a condition, then $s \leq r, w$.

It remains to show that $s$ satisfies requirements (1), (3), and (4) in the definition of $\mathbb{P}$. Regarding (1), it is easy to see that $z$ consists of pairs $\left\langle\alpha, \alpha^{\prime}\right\rangle$ where $\alpha \leq$ $\alpha^{\prime}<\omega_{2}$ and $\alpha \in S$, since this is true of pairs in $x_{r}$ and $x_{w}$ and these properties are 
preserved under isomorphisms. The proof that $z$ consists of nonoverlapping pairs will use requirement (3), so we verify (3) first.

(3) Let $\left\langle\alpha, \alpha^{\prime}\right\rangle \in z$ and $M \in C$ be given, and assume that $M \nsubseteq \nsubseteq \alpha$. We will show that $M \cap\left[\alpha, \alpha^{\prime}\right] \neq \emptyset$ implies that $\alpha$ and $\alpha^{\prime}$ are in $M$, and $M \cap\left[\alpha, \alpha^{\prime}\right]=\emptyset$ implies that $\left\langle\alpha_{M}, \alpha_{M}\right\rangle \in z$.

Case 1: $N \leq M$ and $\left\langle\alpha, \alpha^{\prime}\right\rangle \in x_{r}$. Then $M$ is in $A_{r}$. So we are done since $r$ is a condition.

Case 2: $N \leq M$ and $\left\langle\alpha, \alpha^{\prime}\right\rangle=\sigma_{N, N^{\prime}}\left(\left\langle\alpha_{0}, \alpha_{0}^{\prime}\right\rangle\right)$ for some $\left\langle\alpha_{0}, \alpha_{0}^{\prime}\right\rangle \in x_{w}$ and $N^{\prime} \in$ $A_{r}$ isomorphic to $N$. Since $N \leq M, N^{\prime} \leq M$. If $\alpha^{\prime}<\beta_{M, N^{\prime}}$, then $N^{\prime} \cap \beta_{M, N^{\prime}} \subseteq M$ implies that $\alpha$ and $\alpha^{\prime}$ are in $M$. So assume that $\beta_{M, N^{\prime}} \leq \alpha^{\prime}$.

We claim that $R_{M}\left(N^{\prime}\right) \cap\left(\alpha, \alpha^{\prime}\right]=\emptyset$. Otherwise assume that $\tau$ is in this intersection. Since $r$ is a condition, $\langle\tau, \tau\rangle$ is in $x_{r} \cap N^{\prime}$. Let $\tau_{0}:=\sigma_{N^{\prime}, N}(\tau)$. Then $\left\langle\tau_{0}, \tau_{0}\right\rangle$ is in $x_{r} \cap N$ and hence in $x_{w}$. Since $\sigma_{N^{\prime}, N}$ is order preserving, $\alpha_{0}<\tau_{0} \leq \alpha_{0}^{\prime}$, which contradicts that $w$ is a condition.

If $\alpha<\beta_{M, N^{\prime}} \leq \alpha^{\prime}$, then $\min \left(N^{\prime} \backslash \beta_{M, N^{\prime}}\right)$ is in $R_{M}\left(N^{\prime}\right) \cap\left(\alpha, \alpha^{\prime}\right]$, contradicting the claim. Therefore $\beta_{M, N^{\prime}} \leq \alpha$. If $\xi \in M \cap\left[\alpha, \alpha^{\prime}\right]$, then $\xi \in M \backslash \beta_{M, N^{\prime}}$. So $\min \left(N^{\prime} \backslash \xi\right)$ is in $R_{M}\left(N^{\prime}\right) \cap\left(\alpha, \alpha^{\prime}\right]$, contradicting the claim. So $M \cap\left[\alpha, \alpha^{\prime}\right]=\emptyset$. Since $\alpha \in N^{\prime} \backslash \beta_{M, N^{\prime}}, \alpha_{M}=\min (M \backslash \alpha)$ is in $R_{N^{\prime}}(M)$. Hence $\left\langle\alpha_{M}, \alpha_{M}\right\rangle \in x_{r}$.

Case 3: $M=\sigma_{N, N^{\prime}}(K)$ for some $K \in A_{w}$ and $N^{\prime}$ in $A_{r}$ isomorphic to $N$ and $N^{\prime} \cap\left[\alpha, \alpha^{\prime}\right] \neq \emptyset$. Then $\alpha$ and $\alpha^{\prime}$ are in $N^{\prime}$ by cases 1 and 2. Let $\alpha_{0}:=\sigma_{N^{\prime}, N}(\alpha)$ and $\alpha_{0}^{\prime}:=\sigma_{N^{\prime}, N}\left(\alpha^{\prime}\right)$. Since $s$ satisfies requirement $(5),\left\langle\alpha_{0}, \alpha_{0}^{\prime}\right\rangle$ is in $z \cap N$. Since $z \cap N=x_{w},\left\langle\alpha_{0}, \alpha_{0}^{\prime}\right\rangle \in x_{w}$.

Assume that $M \cap\left[\alpha, \alpha^{\prime}\right] \neq \emptyset$. Applying $\sigma_{N^{\prime}, N}, K \cap\left[\alpha_{0}, \alpha_{0}^{\prime}\right] \neq \emptyset$. Since $w$ is a condition, $\alpha_{0}$ and $\alpha_{0}^{\prime}$ are in $K$. Therefore their images under $\sigma_{N, N^{\prime}}$, namely $\alpha$ and $\alpha^{\prime}$, are in $\sigma_{N, N^{\prime}}(K)=M$.

Now assume that $M \cap\left[\alpha, \alpha^{\prime}\right]=\emptyset$. Applying $\sigma_{N^{\prime}, N}, K \cap\left[\alpha_{0}, \alpha_{0}^{\prime}\right]=\emptyset$. Also $\sigma_{N^{\prime}, N}\left(\alpha_{M}\right)=\sigma_{N^{\prime}, N}(\min (M \backslash \alpha))=\min \left(K \backslash \alpha_{0}\right)=\left(\alpha_{0}\right)_{K}$. Since $w$ is a condition, $\left\langle\left(\alpha_{0}\right)_{K},\left(\alpha_{0}\right)_{K}\right\rangle \in x_{w}$. Therefore $\sigma_{N, N^{\prime}}\left(\left\langle\left(\alpha_{0}\right)_{K},\left(\alpha_{0}\right)_{K}\right\rangle\right)=\left\langle\alpha_{M}, \alpha_{M}\right\rangle$ is in $z$.

Case 4: $M=\sigma_{N, N^{\prime}}(K)$ for some $K \in A_{w}$ and $N^{\prime}$ in $A_{r}$ isomorphic to $N$ and $N^{\prime} \cap\left[\alpha, \alpha^{\prime}\right]=\emptyset$. As $M \in N^{\prime}$, clearly $M \cap\left[\alpha, \alpha^{\prime}\right]=\emptyset$. Since $\alpha_{M}$ exists, $\tau:=$ $\min \left(N^{\prime} \backslash \alpha\right)$ exists. As $N \leq N^{\prime},\langle\tau, \tau\rangle \in z$ by Cases 1 and 2 . If $\tau=\alpha_{M}$ then we are done. Otherwise $\tau<\alpha_{M}$ and $\alpha_{M}=\min (M \backslash \tau)$. Let $\tau_{0}=\sigma_{N^{\prime}, N}(\tau)$. Since $s$ satisfies requirement (5), $\left\langle\tau_{0}, \tau_{0}\right\rangle$ is in $z \cap N=x_{w}$. Also $\min \left(K \backslash \tau_{0}\right)$ is equal to $\sigma_{N^{\prime}, N}(\min (M \backslash$ $\tau)$ ), which is $\sigma_{N^{\prime}, N}\left(\alpha_{M}\right)$. As $w$ is a condition, $\left\langle\sigma_{N^{\prime}, N}\left(\alpha_{M}\right), \sigma_{N^{\prime}, N}\left(\alpha_{M}\right)\right\rangle$ is in $x_{w}$. So the image of this pair under $\sigma_{N, N^{\prime}}$, namely $\left\langle\alpha_{M}, \alpha_{M}\right\rangle$, is in $z$.

Now we show that $z$ consists of nonoverlapping pairs. Suppose that $\left\langle\alpha, \alpha^{\prime}\right\rangle$ and $\left\langle\gamma, \gamma^{\prime}\right\rangle$ are in $z$, and we will prove that it is not the case that $\alpha<\gamma \leq \alpha^{\prime}$. If both pairs are in $x_{r}$ then we are done since $r$ is a condition, so assume not. Suppose for a contradiction that $\alpha<\gamma \leq \alpha^{\prime}$.

Case 1: $\left\langle\alpha, \alpha^{\prime}\right\rangle=\sigma_{N, N^{\prime}}\left(\left\langle\alpha_{0}, \alpha_{0}^{\prime}\right\rangle\right)$ for some $\left\langle\alpha_{0}, \alpha_{0}^{\prime}\right\rangle \in x_{w}$ and $N^{\prime}$ in $A_{r}$ isomorphic to $N$, and $N^{\prime} \cap\left[\gamma, \gamma^{\prime}\right]=\emptyset$. Let $\tau:=\min \left(N^{\prime} \backslash \gamma\right)$, which exists because $\alpha^{\prime} \in N^{\prime}$. Then $\alpha<\tau \leq \alpha^{\prime}$. Since $s$ satisfies requirement (3), $\langle\tau, \tau\rangle$ is in $z \cap N^{\prime}$. 
Let $\tau_{0}:=\sigma_{N^{\prime}, N}(\tau)$. Since $s$ satisfies requirement $(5),\left\langle\tau_{0}, \tau_{0}\right\rangle$ is in $z \cap N=x_{w}$. But then $\alpha_{0}<\tau_{0} \leq \alpha_{0}^{\prime}$, contradicting that $w$ is a condition.

Case 2: $\left\langle\alpha, \alpha^{\prime}\right\rangle=\sigma_{N, N^{\prime}}\left(\left\langle\alpha_{0}, \alpha_{0}^{\prime}\right\rangle\right)$ for some $\left\langle\alpha_{0}, \alpha_{0}^{\prime}\right\rangle \in x_{w}$ and $N^{\prime}$ in $A_{r}$ isomorphic to $N$, and $N^{\prime} \cap\left[\gamma, \gamma^{\prime}\right] \neq \emptyset$. Then by requirement (3), $\gamma$ and $\gamma^{\prime}$ are in $N^{\prime}$. Let $\gamma_{0}=\sigma_{N^{\prime}, N}(\gamma)$ and $\gamma_{0}^{\prime}=\sigma_{N^{\prime}, N}\left(\gamma^{\prime}\right)$. Since $s$ satisfies requirement $(5),\left\langle\gamma_{0}, \gamma_{0}\right\rangle$ is in $z \cap N=x_{w}$. But then $\alpha_{0}<\gamma_{0} \leq \alpha_{0}^{\prime}$, contradicting that $w$ is a condition.

Case 3: $\left\langle\gamma, \gamma^{\prime}\right\rangle=\sigma_{N, N^{\prime}}\left(\left\langle\gamma_{0}, \gamma_{0}^{\prime}\right\rangle\right)$ for some $\left\langle\gamma_{0}, \gamma_{0}^{\prime}\right\rangle \in x_{w}$ and $N^{\prime} \in A_{r}$ isomorphic to $N$. Since $\alpha<\gamma \leq \alpha^{\prime}, N^{\prime} \cap\left[\alpha, \alpha^{\prime}\right] \neq \emptyset$. Since $s$ satisfies requirement (3), $\alpha$ and $\alpha^{\prime}$ are in $N^{\prime}$. Let $\alpha_{0}:=\sigma_{N^{\prime}, N}(\alpha)$ and $\alpha_{0}^{\prime}=\sigma_{N^{\prime}, N}\left(\alpha^{\prime}\right)$. Since $s$ satisfies requirement (5), $\left\langle\alpha_{0}, \alpha_{0}^{\prime}\right\rangle$ is in $z \cap N=x_{w}$. But then $\alpha_{0}<\gamma_{0} \leq \alpha_{0}^{\prime}$, contradicting that $w$ is a condition.

Since at least one of $\left\langle\alpha, \alpha^{\prime}\right\rangle$ and $\left\langle\gamma, \gamma^{\prime}\right\rangle$ is not in $x_{r}$, these cases cover all possibilities.

(4) Let $\zeta \in R_{C}$ be given, and we will show that $\langle\zeta, \zeta\rangle \in z$. By Proposition 5.2, $\zeta$ is in the set

$R_{A_{r}} \cup\left\{\min (K \backslash \xi): K \in C, \xi \in R_{A_{r}}\right\} \cup\left\{\sigma_{N, N^{\prime}}(\tau): N^{\prime} \in A, N \cong N^{\prime}, \tau \in R_{A_{w}}\right\}$.

Let $\zeta \in R_{C}$ be given, and we will show that $\langle\zeta, \zeta\rangle \in z$.

Case 1: $\zeta \in R_{A_{r}}$. Since $r$ is a condition, $\langle\zeta, \zeta\rangle \in x_{r}$.

Case 2: $\zeta=\sigma_{N, N^{\prime}}(\tau)$ for some $\tau \in R_{A_{w}}$ and $N^{\prime} \in A_{r}$ isomorphic to $N$. Since $w$ is a condition, $\langle\tau, \tau\rangle \in x_{w}$. So $\sigma_{N, N^{\prime}}(\langle\tau, \tau\rangle)=\langle\zeta, \zeta\rangle$ is in $z$ by definition.

Case 3: For some $K \in C$ and $\xi \in R_{A_{r}}, \zeta=\min (K \backslash \xi)$. Then $\langle\xi, \xi\rangle \in x_{r}$. First assume that $K \in A_{r}$. Then since $r$ is a condition, $\langle\zeta, \zeta\rangle \in x_{r}$.

Secondly assume that $K=\sigma_{N, N^{\prime}}(L)$ for some $L \in A_{w}$ and $N^{\prime} \in A_{r}$ isomorphic to $N$. Let $\tau:=\min \left(N^{\prime} \backslash \xi\right)$. Then $\zeta=\min (K \backslash \tau)$. Since $r$ is a condition, $\langle\tau, \tau\rangle \in x_{r}$. Let $\tau_{0}:=\sigma_{N^{\prime}, N}(\tau)$. Then $\left\langle\tau_{0}, \tau_{0}\right\rangle \in x_{r} \cap N=x_{w}$. Since $\zeta=\min (K \backslash \tau)$, applying $\sigma_{N^{\prime}, N}$ we get that $\zeta_{0}:=\sigma_{N^{\prime}, N}(\zeta)=\min \left(L \backslash \tau_{0}\right)$. Since $w$ is a condition, $\left\langle\zeta_{0}, \zeta_{0}\right\rangle$ is in $x_{w}$. Therefore $\sigma_{N, N^{\prime}}\left(\left\langle\zeta_{0}, \zeta_{0}\right\rangle\right)=\langle\zeta, \zeta\rangle$ is in $z$.

Proposition 7.3. The forcing poset $\mathbb{P}$ is an $(S, \mathcal{Y})$ coherent adequate type forcing.

Proof. Let $(x, A)$ be a condition in $\mathbb{P}$, and we will show that $(x, A)$ satisfies properties $(\mathrm{I})-(\mathrm{V})$ in the definition of an $(S, \mathcal{Y})$ coherent adequate type forcing.

(I) is immediate, and (V) follows from Proposition 7.2. (II) By the definition of $\mathbb{P}, A$ is a finite coherent adequate subset of $\mathcal{Y}$. If $M$ and $N$ are in $A$ and $\zeta \in R_{M}(N)$, then $\langle\zeta, \zeta\rangle \in x$. This implies that $\zeta \in S$. Therefore $A$ is $(S)$ adequate.

(III) Let $(y, B) \leq(x, A)$, and assume that $N$ and $N^{\prime}$ are isomorphic in $B$ with $(x, A) \in N$. Let $\sigma:=\sigma_{N, N^{\prime}}$. We will show that $\sigma((x, A))$ is in $\mathbb{P}$ and $(y, B) \leq$ $\sigma((x, A))$. All the properties of being in $\mathbb{P}$ described in Definition 7.1 are first order definable in $H\left(\omega_{2}\right)$ without parameters, except for the requirements on membership in $S$ and $\mathcal{Y}$. Therefore as $N$ and $N^{\prime}$ are elementary in $H\left(\omega_{2}\right)$ and $\sigma$ preserves membership in $S$ and $\mathcal{Y},(x, A)$ being in $\mathbb{P}$ implies that $\sigma((x, A))$ is in $\mathbb{P}$. Also $\sigma((x, A))=(\sigma[x], \sigma[A])$, and since $(y, B)$ is a condition with $x \subseteq y$ and $A \subseteq B$, $\sigma[x] \subseteq y$ and $\sigma[A] \subseteq B$. So $(y, B) \leq \sigma((x, A))$. 
(IV) Suppose that $M_{0}, \ldots, M_{n}$ are isomorphic sets in $\mathcal{Y}$ such that $\left\{M_{0}, \ldots, M_{n}\right\}$ is $(S)$ coherent adequate and $(x, A) \in M_{0} \cap \cdots \cap M_{n}$. Let $C=A \cup\left\{M_{0}, \ldots, M_{n}\right\}$. By Lemma 1.17, $C$ is coherent adequate.

Let $M$ and $N$ be in $C$. If $M$ is in $\left\{M_{0}, \ldots, M_{n}\right\}$ and $N$ is in $A$, then $N \in M$. Hence $R_{N}(M)$ and $R_{M}(N)$ are empty. It follows that $R_{C}=R_{A} \cup\left\{R_{M_{i}}\left(M_{j}\right)\right.$ : $i, j \leq n\}$. In particular, $R_{C} \subseteq S$. So $C$ is $(S)$ coherent adequate.

Define

$$
z=y \cup\left\{\sigma_{K, L}(a): K, L \in C, K \cong L, a \in y \cap K\right\},
$$

where $y=x \cup\left\{\langle\zeta, \zeta\rangle: \zeta \in R_{C} \backslash R_{A}\right\}$. By Lemma 4.1, $(z, C)$ is closed. We will show that $(z, C)$ is condition. Then clearly $(z, C) \leq(x, A)$ and $M_{0}, \ldots, M_{k} \in C$, which finishes the proof.

First we claim that $z$ is equal to

$$
z^{\prime}:=x \cup\left\{\sigma_{M_{i}, M_{j}}(\langle\zeta, \zeta\rangle): i, j \leq n, \zeta \in\left(R_{C} \cap M_{i}\right) \backslash R_{A}\right\} .
$$

Obviously $z^{\prime} \subseteq z$.

For the other direction, first let us prove that $y \subseteq z^{\prime}$. By definition, $x \subseteq z^{\prime}$. Now consider $\zeta \in R_{C} \backslash R_{A}$. Then $\zeta \in R_{M_{i}}\left(M_{j}\right)$ for some $i, j \leq n$. Hence $\langle\zeta, \zeta\rangle=$ $\sigma_{M_{j}, M_{j}}(\langle\zeta, \zeta\rangle)$ is in $z^{\prime}$.

Secondly assume that $b=\sigma_{K, L}(a)$ where $K, L \in C, K \cong L$, and $a \in y \cap K$. Then either $K, L \in A$ or $K, L \in\left\{M_{0}, \ldots, M_{n}\right\}$. In the former case, $a$ being in $K$ implies that $a$ is not in $R_{C} \backslash R_{A}$, since every member of $R_{C} \backslash R_{A}$ lies above every ordinal in $K \cap \omega_{2}$. Hence $a$ is in $x$. So $b \in x$ since $(x, A)$ is a condition.

Assume that $K, L \in\left\{M_{0}, \ldots, M_{n}\right\}$. If $a \in x$ then $a \in K \cap L$, so $b=\sigma_{K, L}(a)=a$. So $b \in y$, and hence $b \in z^{\prime}$. Otherwise $a=\langle\zeta, \zeta\rangle$ where $\zeta$ is in $R_{C} \backslash R_{A}$, and then by definition $b$ is in $z^{\prime}$.

We have shown that $z=z^{\prime}$. Note that if $\langle\alpha, \alpha\rangle$ is in $z \backslash x$, then for all $K \in A$ and $\left\langle\gamma, \gamma^{\prime}\right\rangle$ in $x, \sup \left(K \cap \omega_{2}\right)$ and $\gamma^{\prime}$ are below $\alpha$. For suppose $\langle\alpha, \alpha\rangle=\sigma_{M_{i}, M_{j}}(\langle\zeta, \zeta\rangle)$ for some $\zeta \in\left(R_{C} \cap M_{i}\right) \backslash R_{A}$. Then $\zeta \in R_{M_{k}}\left(M_{l}\right)$ for some $k, l \leq n$. So $\beta_{M_{k}, M_{l}} \leq \zeta$. Since $(x, A) \in M_{k} \cap M_{l}$, any ordinals in $\omega_{2}$ definable from $(x, A)$ are in $\beta_{M_{k}, M_{l}}$ and hence are below $\zeta$. If $\sigma_{M_{i}, M_{j}}(\zeta)=\zeta$ then we are done. Otherwise $\beta_{M_{i}, M_{j}} \leq \zeta$, so clearly $\beta_{M_{i}, M_{j}} \leq \sigma_{M_{i}, M_{j}}(\zeta)$. And as $(x, A) \in M_{i} \cap M_{j}$, again any ordinals definable from $(x, A)$ are below $\sigma_{M_{i}, M_{j}}(\zeta)$.

We now verify that $(z, C)$ satisfies properties $(1)-(5)$ in the definition of $\mathbb{P}$. We already noted that $C$ is a coherent adequate subset of $\mathcal{Y}$ and $(z, C)$ is closed. Hence properties (2) and (5) holds. Also (4) is immediate.

(1) Since isomorphisms preserve membership in $S$, easily $z$ consists of pairs of the form $\left\langle\alpha, \alpha^{\prime}\right\rangle$ where $\alpha \leq \alpha^{\prime}<\omega_{2}$ and $\alpha \in S$. Pairs which lie in $x$ are nonoverlapping, and pairs lying in $z \backslash x$ are nonoverlapping because the first and second components in such a pair are equal. Consider $\left\langle\alpha, \alpha^{\prime}\right\rangle \in x$ and $\sigma_{M_{i}, M_{j}}(\zeta)$ where $i, j \leq n$ and $\zeta \in\left(R_{C} \cap M_{i}\right) \backslash R_{A}$. By the comment above, $\alpha^{\prime}<\sigma_{M_{i}, M_{j}}(\zeta)$. Hence $\left\langle\alpha, \alpha^{\prime}\right\rangle$ and $\sigma_{M_{i}, M_{j}}(\langle\zeta, \zeta\rangle)$ do not overlap.

(3) Let $\left\langle\alpha, \alpha^{\prime}\right\rangle \in z$ and $K \in C$ with $K \cap \omega_{2} \nsubseteq \alpha$. If $\left\langle\alpha, \alpha^{\prime}\right\rangle \in x$ and $K \in A$, then we are done. If $\left\langle\alpha, \alpha^{\prime}\right\rangle \in x$ and $K \in\left\{M_{0}, \ldots, M_{n}\right\}$, then $\alpha$ and $\alpha^{\prime}$ are in $K$.

Suppose that $\left\langle\alpha, \alpha^{\prime}\right\rangle$ is equal to $\sigma_{M_{i}, M_{j}}(\langle\zeta, \zeta\rangle)$ for some $i, j \leq n$ and $\zeta \in\left(R_{C} \cap\right.$ $\left.M_{i}\right) \backslash R_{A}$. Then $\alpha=\alpha^{\prime}$. If $K \in A$, then by the comment above, $\sup \left(K \cap \omega_{2}\right)<\alpha$, contradicting our assumption. Therefore $K \in\left\{M_{0}, \ldots, M_{n}\right\}$. If $\alpha \in K$ then we are done, so assume not. Then $\alpha \in M_{j} \backslash K$. Since $K \cap \beta_{K, M_{j}}=M_{j} \cap \beta_{K, M_{j}}$, 
$\beta_{K, M_{j}} \leq \alpha$. So $\alpha \in M_{j} \backslash \beta_{K, M_{j}}$. It follows that $\alpha_{K}=\min (K \backslash \alpha)$ is in $R_{M_{j}}(K)$. By definition of $z,\left\langle\alpha_{K}, \alpha_{K}\right\rangle$ is in $z$.

It remains to show that $\mathbb{P}$ forces that $\dot{C}_{S}$ is a club. For unboundedness, given a condition $p$ and an ordinal $\gamma$, choose $\beta$ in $S$ larger than all ordinals appearing in pairs of $p$ and all suprema of models appearing in $p$ intersected with $\omega_{2}$. Then $\left(x_{p} \cup\{\langle\beta, \beta\rangle\}, A_{p}\right)$ is a condition which forces that $\dot{C}_{S}$ is not a subset of $\gamma$.

The proof of the closure of $\dot{C}_{S}$ is similar to the argument from [4, except that we have the new problem of needing to close under isomorphisms when adding something to a condition. This problem is dealt with by the next lemma.

Lemma 7.4. Let $(x, A)$ be a condition and let $\left\langle\alpha, \alpha^{\prime}\right\rangle$ be a pair such that $\alpha \leq \alpha^{\prime}<$ $\omega_{2}$ and $\alpha \in S$. Assume:

(1) $\left\langle\alpha, \alpha^{\prime}\right\rangle$ does not overlap any pair in $x$;

(2) if $N \in A$ and $N \cap \omega_{2} \nsubseteq \alpha$, then $N \cap\left[\alpha, \alpha^{\prime}\right] \neq \emptyset$ implies that $\alpha$ and $\alpha^{\prime}$ are in $N$, and $N \cap\left[\alpha, \alpha^{\prime}\right]=\emptyset$ implies that $\left\langle\alpha_{N}, \alpha_{N}\right\rangle \in x_{p}$.

Let $z$ be the set

$$
x \cup\left\{\left\langle\alpha, \alpha^{\prime}\right\rangle\right\} \cup\left\{\sigma_{N, N^{\prime}}\left(\left\langle\alpha, \alpha^{\prime}\right\rangle\right): N, N^{\prime} \in A_{r}, N \cong N^{\prime},\left\langle\alpha, \alpha^{\prime}\right\rangle \in N\right\} .
$$

Then $(z, A)$ is a condition below $(x, A)$ and $\left\langle\alpha, \alpha^{\prime}\right\rangle \in z$.

Proof. We will prove that $(z, A)$ is a condition. Then it is clear that $(z, A) \leq(x, A)$ and $\left\langle\alpha, \alpha^{\prime}\right\rangle \in x$. Properties (2) and (4) in the definition of $\mathbb{P}$ are immediate.

(5) Let $y:=x \cup\left\{\left\langle\alpha, \alpha^{\prime}\right\rangle\right\}$. Since $(x, A)$ is closed, it is easy to see that $z$ is equal to

$$
y \cup\left\{\sigma_{N, N^{\prime}}(a): N, N^{\prime} \in A, N \cong N^{\prime}, a \in y \cap N\right\} .
$$

By Lemma $4.1,(z, A)$ is closed.

(3) Consider a pair $\left\langle\alpha_{1}, \alpha_{1}^{\prime}\right\rangle=\sigma_{N, N^{\prime}}\left(\left\langle\alpha, \alpha^{\prime}\right\rangle\right)$, where $N$ and $N^{\prime}$ are isomorphic in $A$ and $\left\langle\alpha, \alpha^{\prime}\right\rangle \in N$. Let $M \in A$ be given such that $M \cap \omega_{2} \nsubseteq \alpha_{1}$.

We claim that $R_{A} \cap\left(\alpha_{1}, \alpha_{1}^{\prime}\right]=\emptyset$. Suppose for a contradiction that $\zeta$ is in this intersection. Then $\langle\zeta, \zeta\rangle \in x$. So $\sigma_{N^{\prime}, N}(\langle\zeta, \zeta\rangle) \in x$. But this last pair overlaps with $\left\langle\alpha, \alpha^{\prime}\right\rangle$, which contradicts our assumptions.

Case 1: $M \cap\left[\alpha_{1}, \alpha_{1}^{\prime}\right] \neq \emptyset$. We will show that $\alpha_{1}$ and $\alpha_{1}^{\prime}$ are in $M$. Suppose that there is $\theta \in M \cap\left[\alpha_{1}, \alpha_{1}^{\prime}\right]$ such that $\beta_{M, N^{\prime}} \leq \theta$. Let $\zeta:=\min \left(N^{\prime} \backslash \theta\right)$. Then $\zeta$ is in $R_{A}$ and $\alpha_{1}<\zeta \leq \alpha_{1}^{\prime}$, contradicting the claim above. Therefore any ordinal in the nonempty intersection $M \cap\left[\alpha_{1}, \alpha_{1}^{\prime}\right]$ is strictly below $\beta_{M, N^{\prime}}$. In particular, $\alpha_{1}<\beta_{M, N^{\prime}}$.

Assume that $N^{\prime} \leq M$. If $\beta_{M, N^{\prime}} \leq \alpha_{1}^{\prime}$, then $\min \left(N^{\prime} \backslash \beta_{M, N^{\prime}}\right)$ is in $R_{A} \cap\left(\alpha_{1}, \alpha_{1}^{\prime}\right.$, contradicting the claim above. So $\alpha_{1}^{\prime}<\beta_{M, N^{\prime}}$. Then $\alpha_{1}$ and $\alpha_{1}^{\prime}$ are in $N^{\prime} \cap \beta_{M, N^{\prime}}$ and hence in $M$.

Now assume that $M<N^{\prime}$. Fix $N^{*}$ in $A$ isomorphic to $N^{\prime}$ such that $M \in N^{*}$. Since $M \in N^{*}, \beta_{M, N^{\prime}} \leq \beta_{N^{*}, N^{\prime}}$. In particular, $\alpha_{1}<\beta_{N^{*}, N^{\prime}}$. If $\beta_{N^{*}, N^{\prime}} \leq \alpha_{1}^{\prime}$, then $\min \left(N^{\prime} \backslash \beta_{N^{*}, N^{\prime}}\right)$ is in $R_{A} \cap\left(\alpha_{1}, \alpha_{1}^{\prime}\right]$, contradicting the claim. Therefore $\alpha_{1}^{\prime}<\beta_{N^{*}, N^{\prime}}$. It follows that $\alpha_{1}$ and $\alpha_{1}^{\prime}$ are in $N^{*}$.

By Lemma 1.13, $\left\langle\alpha, \alpha^{\prime}\right\rangle=\sigma_{N^{\prime}, N}\left(\left\langle\alpha_{1}, \alpha_{1}^{\prime}\right\rangle\right)$ is equal to $\sigma_{N^{*}, N}\left(\left\langle\alpha_{1}, \alpha_{1}^{\prime}\right\rangle\right)$. Since $M \cap$ $\left[\alpha_{1}, \alpha_{1}^{\prime}\right] \neq \emptyset$, applying $\sigma_{N^{*}, N}$ we get that $\sigma_{N^{*}, N}(M) \cap\left[\alpha, \alpha^{\prime}\right] \neq \emptyset$. As $\sigma_{N^{*}, N}(M) \in A$, our assumptions imply that $\alpha$ and $\alpha^{\prime}$ are in $\sigma_{N^{*}, N}(M)$. Applying $\sigma_{N, N^{*}}$, we get that $\alpha_{1}$ and $\alpha_{1}^{\prime}$ are in $M$. 
Case 2: $M \cap\left[\alpha_{1}, \alpha_{1}^{\prime}\right]=\emptyset$. Let $\beta:=\min \left(M \backslash \alpha_{1}\right)$, and we will show that $\langle\beta, \beta\rangle \in z$. Note that $\beta$ equals $\min \left(M \backslash \alpha_{1}^{\prime}\right)$. So if $\beta_{M, N^{\prime}} \leq \alpha_{1}^{\prime}$, then $\beta \in R_{A}$ and hence $\langle\beta, \beta\rangle \in x$. So assume that $\alpha_{1}^{\prime}<\beta_{M, N^{\prime}}$. Since $M$ does not contain $\alpha_{1}$, we must have that $M<N^{\prime}$. If $\beta_{M, N^{\prime}} \leq \beta$, then $\beta=\min \left(M \backslash \beta_{M, N^{\prime}}\right)$. So $\beta \in R_{A}$ and again $\langle\beta, \beta\rangle \in x$. Assume that $\beta<\beta_{M, N^{\prime}}$. Since $M<N^{\prime}$, it follows that $\beta \in M \cap N^{\prime}$.

Fix $N^{*}$ in $A$ which is isomorphic to $N^{\prime}$ with $M \in N^{*}$. Since $\beta \in N^{\prime} \cap N^{*}$, $\beta<\beta_{N^{\prime}, N^{*}}$. So $\alpha_{1}$ and $\alpha_{1}^{\prime}$ are in $N^{*}$. By Lemma 1.13, $\sigma_{N^{*}, N}\left(\left\langle\alpha_{1}, \alpha_{1}^{\prime}\right\rangle\right)=$ $\sigma_{N^{\prime}, N}\left(\left\langle\alpha_{1}, \alpha_{1}^{\prime}\right\rangle\right)=\left\langle\alpha, \alpha^{\prime}\right\rangle$. Since $M \cap\left[\alpha_{1}, \alpha_{1}^{\prime}\right]=\emptyset$, applying $\sigma_{N^{*}, N}$ we get that $\sigma_{N^{*}, N}(M) \cap\left[\alpha, \alpha^{\prime}\right]=\emptyset$. Let $\pi:=\min \left(\sigma_{N^{*}, N}(M) \backslash \alpha\right)$. As $\sigma_{N^{*}, N}(M) \in A$, our assumptions imply that $\langle\pi, \pi\rangle \in x$. But $\beta=\min \left(M \backslash \alpha_{1}\right)$, so applying $\sigma_{N^{*}, N}$ we get that $\sigma_{N^{*}, N}(\beta)=\min \left(\sigma_{N^{*}, N}(M) \backslash \alpha\right)=\pi$. Hence $\left\langle\sigma_{N^{*}, N}(\beta), \sigma_{N^{*}, N}(\beta)\right\rangle$ is in $x$. Since $(x, A)$ is closed, $\langle\beta, \beta\rangle \in x$.

(1) It is immediate that the pairs of $z$ are of the correct form. We will show that they are nonoverlapping. This is true by assumption for all pairs in $x \cup\left\{\left\langle\alpha, \alpha^{\prime}\right\rangle\right\}$.

Case 1: Suppose that $\left\langle\gamma, \gamma^{\prime}\right\rangle \in x$ and $\left\langle\alpha_{1}, \alpha_{1}^{\prime}\right\rangle=\sigma_{N, N^{\prime}}\left(\left\langle\alpha, \alpha^{\prime}\right\rangle\right)$, where $N$ and $N^{\prime}$ are isomorphic in $A$ and $\left\langle\alpha, \alpha^{\prime}\right\rangle \in N$. First assume that $N^{\prime} \cap\left[\gamma, \gamma^{\prime}\right] \neq \emptyset$. Then $\gamma$ and $\gamma^{\prime}$ are in $N^{\prime}$. Hence any overlap between $\left\langle\alpha_{1}, \alpha_{1}^{\prime}\right\rangle$ and $\left\langle\gamma, \gamma^{\prime}\right\rangle$ translates to an overlap between $\left\langle\alpha, \alpha^{\prime}\right\rangle$ and $\sigma_{N^{\prime}, N}\left(\left\langle\gamma, \gamma^{\prime}\right\rangle\right)$. As $\sigma_{N^{\prime}, N}\left(\left\langle\gamma, \gamma^{\prime}\right\rangle\right) \in x$, this would contradict our assumptions.

Assume that $N^{\prime} \cap\left[\gamma, \gamma^{\prime}\right]=\emptyset$. Then the only possible overlap between $\left\langle\gamma, \gamma^{\prime}\right\rangle$ and $\left\langle\alpha_{1}, \alpha_{1}^{\prime}\right\rangle$ would be if $\alpha_{1}<\gamma \leq \gamma^{\prime}<\alpha_{1}^{\prime}$. Letting $\zeta:=\min \left(N^{\prime} \backslash \gamma\right)$, we have that $\langle\zeta, \zeta\rangle \in x$ and $\alpha_{1}<\zeta \leq \alpha_{1}^{\prime}$. But now we get a contradiction exactly as in the previous paragraph.

Case 2: Suppose that $\left\langle\alpha_{1}, \alpha_{1}^{\prime}\right\rangle=\sigma_{N, N^{\prime}}\left(\left\langle\alpha, \alpha^{\prime}\right\rangle\right)$ and $\left\langle\alpha_{2}, \alpha_{2}^{\prime}\right\rangle=\sigma_{M, M^{\prime}}\left(\left\langle\alpha, \alpha^{\prime}\right\rangle\right)$, where $N, N^{\prime}, M, M^{\prime}$ are in $A, N \cong N^{\prime}$, and $M \cong M^{\prime}$. Suppose for a contradiction that $\alpha_{1}<\alpha_{2} \leq \alpha_{1}^{\prime}$. Then $M^{\prime} \cap\left[\alpha_{1}, \alpha_{1}^{\prime}\right] \neq \emptyset$. By property (3) of $(z, C)$, this implies that $\alpha_{1}$ and $\alpha_{1}^{\prime}$ are in $M^{\prime}$. Since these ordinals are also in $N^{\prime}, \alpha_{1}^{\prime}<\beta_{M^{\prime}, N^{\prime}}$.

First assume that $M$ and $N$ are isomorphic. As $\alpha$ and $\alpha^{\prime}$ are in $M \cap N$, $\sigma_{M, N}(\alpha)=\alpha$ and $\sigma_{M, N}\left(\alpha^{\prime}\right)=\alpha^{\prime}$. Since $\alpha_{1}<\alpha_{2} \leq \alpha_{1}^{\prime}<\beta_{M^{\prime}, N^{\prime}}$, these three ordinals are in $M^{\prime} \cap N^{\prime}$, so $\sigma_{M^{\prime}, N^{\prime}}$ fixes them. In particular, we have that $\alpha_{1}=$ $\sigma_{N, N^{\prime}}(\alpha)=\sigma_{M^{\prime}, N^{\prime}}\left(\sigma_{M, M^{\prime}}\left(\sigma_{N, M}(\alpha)\right)\right)=\sigma_{M^{\prime}, N^{\prime}}\left(\sigma_{M, M^{\prime}}(\alpha)\right)=\sigma_{M^{\prime}, N^{\prime}}\left(\alpha_{2}\right)=\alpha_{2}$. But this contradicts that $\alpha_{1}<\alpha_{2}$.

Assume that $M<N$. Fix $N^{*}$ in $A$ isomorphic to $N$ with $M \in N^{*}$. Then $\alpha$ and $\alpha^{\prime}$ are in $N \cap N^{*}$. So $\sigma_{N, N^{\prime}}$ and $\sigma_{N^{*}, N^{\prime}}$ agree on $\alpha$ and $\alpha^{\prime}$ by Lemma 1.13. Let $L:=\sigma_{N^{*}, N^{\prime}}(M)$, which is in $A$ and satisfies that $\sigma_{M, L}=\sigma_{N^{*}, N^{\prime}} \uparrow M$. In particular, $\sigma_{M, L}\left(\left\langle\alpha, \alpha^{\prime}\right\rangle\right)=\sigma_{N^{*}, N^{\prime}}\left(\left\langle\alpha, \alpha^{\prime}\right\rangle\right)=\sigma_{N, N^{\prime}}\left(\left\langle\alpha, \alpha^{\prime}\right\rangle\right)=\left\langle\alpha_{1}, \alpha_{1}^{\prime}\right\rangle$. So $\left\langle\alpha_{1}, \alpha_{1}^{\prime}\right\rangle=\sigma_{M, L}\left(\left\langle\alpha, \alpha^{\prime}\right\rangle\right)$ and $\left\langle\alpha_{2}, \alpha_{2}^{\prime}\right\rangle=\sigma_{M, M^{\prime}}\left(\left\langle\alpha, \alpha^{\prime}\right\rangle\right)$, and thus we have reduced the current situation to the case considered in the previous paragraph. A symmetric argument works when $N<M$.

Note the following consequence of the lemma: if $(x, A)$ is a condition and $\left\langle\beta, \beta^{\prime}\right\rangle \in$ $x$, then there is $(y, A) \leq(x, A)$ such that $\langle\beta, \beta\rangle \in y$. Also the lemma implies that for all $p$, there is $q \leq p$ such that for all $\left\langle\beta, \beta^{\prime}\right\rangle \in x_{q},\langle\beta, \beta\rangle \in x_{q}$. For the process of adding $\langle\beta, \beta\rangle$ to $p$ described in the lemma adds only pairs of the form $\langle\gamma, \gamma\rangle$, where for some $\gamma^{\prime},\left\langle\gamma, \gamma^{\prime}\right\rangle \in x_{p}$. So repeating finitely many times we can close the condition under this requirement. 
Proposition 7.5. The forcing poset $\mathbb{P}$ forces that $\dot{C}_{S}$ is a club.

Proof. Suppose that $p$ forces that $\alpha$ is a limit point of $\dot{C}_{S}$. We will find a condition below $p$ which forces that $\alpha \in \dot{C}_{S}$. If $\left\langle\alpha, \alpha^{\prime}\right\rangle \in x_{p}$ for some $\alpha^{\prime}$, then $p$ forces that $\alpha \in \dot{C}_{S}$ and we are done. So assume not. Then for all $\left\langle\xi, \xi^{\prime}\right\rangle$ in $x_{p}$, either $\xi \leq \xi^{\prime}<\alpha$ or $\alpha<\xi \leq \xi^{\prime}$.

Let $A_{0}:=\left\{K \in A_{p}: \sup \left(K \cap \omega_{2}\right)<\alpha\right\}, A_{1}:=\left\{K \in A_{p}: \sup (K \cap \alpha)<\right.$ $\left.\alpha, K \cap \omega_{2} \nsubseteq \subseteq \alpha\right\}$, and $A_{2}:=\left\{K \in A_{p}: \sup (K \cap \alpha)=\alpha\right\}$. Note that for all $M$ and $N$ in $A_{2}, \alpha$ is a limit point of both $M$ and $N$ and therefore $\alpha<\beta_{M, N}$.

Case 1: $\operatorname{cf}(\alpha)=\omega_{1}$. Extending $p$ if necessary, we may assume that there is $M \in$ $A_{p}$ such $\alpha \in M$. Then $M \cap \alpha$ is bounded below $\alpha$. Since $p$ forces that $\alpha$ is a limit point of $\dot{C}_{S}$, we can fix $q \leq p$ such that for some $\gamma$ and $\gamma^{\prime}, \sup (M \cap \alpha)<\gamma \leq \gamma^{\prime}<\alpha$ and $\left\langle\gamma, \gamma^{\prime}\right\rangle \in x_{q}$. Then $M \cap\left[\gamma, \gamma^{\prime}\right]=\emptyset$ and $\min (M \backslash \gamma)=\alpha$. So $\langle\alpha, \alpha\rangle$ is in $x_{q}$ since $q$ is a condition.

Case 2: $\operatorname{cf}(\alpha)=\omega$. Since $p$ forces that $\alpha$ is a limit point of $\dot{C}_{S}$, fix $t \leq p$ satisfying:

(a) there is $\gamma$ and $\gamma^{\prime}$ satisfying that $\gamma \leq \gamma^{\prime}<\alpha,\left\langle\gamma, \gamma^{\prime}\right\rangle \in x_{t}$, and for all $K \in A_{0} \cup A_{1}, \sup (K \cap \alpha)<\gamma$

(b) $\gamma$ is the largest such ordinal;

(c) for all pairs $\left\langle\beta, \beta^{\prime}\right\rangle$ in $x_{t},\langle\beta, \beta\rangle$ is in $t$.

Let $q:=\left(x_{t}, A_{p}\right)$. Then $q$ is a condition and $q \leq p$. If $\left\langle\alpha, \alpha^{\prime}\right\rangle \in x_{q}$ for some $\alpha^{\prime}$ then we are done, so assume not. It follows that $\langle\alpha, \alpha\rangle$ does not overlap any pair in $x_{q}$ since $\alpha$ is forced to be a limit point of $\dot{C}_{S}$. Also by the maximality of $\gamma$, the pair $\langle\gamma, \alpha\rangle$ does not overlap any pair in $x_{q}$. For all $K \in A_{1}, K \cap\left[\gamma, \gamma^{\prime}\right]=\emptyset$, and clearly $\alpha_{K}=\gamma_{K}$. Hence $\left\langle\alpha_{K}, \alpha_{K}\right\rangle \in x_{q}$. In particular, for all $K \in A_{1}, \alpha<\alpha_{K}$.

Subcase 2a: $A_{2}=\emptyset$. We will use Lemma 7.4 to show we can add $\langle\gamma, \alpha\rangle$ to $q$, contradicting that $\alpha$ is forced to be a limit point of $\dot{C}_{S}$. By the choice of $\gamma, \gamma \in S$ and $\langle\gamma, \alpha\rangle$ does not overlap any pair in $x_{q}$. By the case assumption, if $K \in A_{p}$ and $K \cap \omega_{2} \nsubseteq \not \gamma$, then $K \in A_{1}$. By the choice of $\gamma$ and the comments above, $K \cap[\gamma, \alpha]=\emptyset$ and $\left\langle\alpha_{K}, \alpha_{K}\right\rangle \in x_{q}$. By Lemma 7.4 there is an extension of $q$ which contains $\langle\gamma, \alpha\rangle$.

Subcase 2b: $A_{2} \neq \emptyset$ and there exists $M \in A_{2}$ such that $\sup \left(M \cap \omega_{2}\right)=\alpha$. We apply Lemma 7.4 to show that we can add $\langle\alpha, \alpha\rangle$. Note that $\alpha \in S$ since $M \in \mathcal{Y}$. And $\langle\alpha, \alpha\rangle$ does not overlap any pair in $x_{q}$ as noted above. Let $N \in A_{q}$ be given such that $N \cap \omega_{2} \nsubseteq \nsubseteq \alpha$ and $\alpha \notin N$. Then $N \notin A_{0}$. If $N \in A_{1}$ then we already know that $\left\langle\alpha_{N}, \alpha_{N}\right\rangle \in x_{q}$. Suppose that $N \in A_{2}$. Then $\alpha<\beta_{M, N}$ as pointed out above. In particular, $\alpha=\sup \left(M \cap \beta_{M, N}\right)$. Since $\alpha \notin N$, it is not the case that $M<N$. As $\alpha$ is a limit point of $N \cap \beta_{M, N}$ not in $M$, likewise $N$ cannot be below $M$. Therefore $M \sim N$. Since $\alpha_{N} \notin M, \beta_{M, N} \leq \alpha_{N}$. So $\alpha<\beta_{M, N} \leq \alpha_{N}$. It follows that $\alpha_{N}=\min \left(N \backslash \beta_{M, N}\right)$. So $\alpha_{N} \in R_{M}(N)$. Therefore $\left\langle\alpha_{N}, \alpha_{N}\right\rangle \in x_{q}$.

Subcase 2c: $A_{2} \neq \emptyset$ and for all $M \in A_{2}, M \cap \omega_{2} \nsubseteq \not \alpha$. Let $M$ be a member of $A_{2}$ satisfying that for all $K \in A_{2}$, (i) $M \cap \omega_{1} \leq K \cap \omega_{1}$, and (ii) if $M \cap \omega_{1}=K \cap \omega_{1}$ then $\alpha_{M} \leq \alpha_{K}$.

First assume that there is $\left\langle\beta, \beta^{\prime}\right\rangle$ in $x_{q}$ with $\alpha \leq \beta \leq \alpha_{M}$. Then $\beta_{M}=\alpha_{M}$. By our assumptions, $\beta$ cannot equal $\alpha$, so $\alpha<\beta$. By the choice of $q,\langle\beta, \beta\rangle \in x_{q}$. 
Therefore $\left\langle\alpha_{M}, \alpha_{M}\right\rangle \in x_{q}$. In particular, $\alpha_{M} \in S \cap M$. By the definition of $\mathcal{Y}$, $\sup \left(M \cap \alpha_{M}\right)=\alpha \in S$.

We apply Lemma 7.4 to show that we can add $\langle\alpha, \alpha\rangle$. The pair $\langle\alpha, \alpha\rangle$ does not overlap any pair in $x_{q}$. Let $N \in A_{q}$ be given such that $N \cap \omega_{2} \nsubseteq \alpha$ and $\alpha \notin N$. We will show that $\left\langle\alpha_{N}, \alpha_{N}\right\rangle \in x_{q}$. Obviously $N \notin A_{0}$. And if $N \in A_{1}$, then we already know that $\left\langle\alpha_{N}, \alpha_{N}\right\rangle \in x_{q}$. Let $N \in A_{2}$. Then by (i), $M \leq N$. Recall that $\alpha<\beta_{M, N}$. Since $\alpha \notin N$ and $\alpha$ is a limit point of $M \cap \beta_{M, N}$, it is not possible that $M<N$. So $M \cap \omega_{1}=N \cap \omega_{1}$. By (ii), $\alpha_{M} \leq \alpha_{N}$. But then $\alpha<\beta \leq \alpha_{N}$, so $\beta_{N}=\alpha_{N}$. Since $q$ is a condition, $\left\langle\beta_{N}, \beta_{N}\right\rangle \in x_{q}$. So $\left\langle\alpha_{N}, \alpha_{N}\right\rangle \in x_{q}$.

Now assume that there is no such pair $\left\langle\beta, \beta^{\prime}\right\rangle$ in $x_{q}$. In particular, $\left\langle\alpha_{M}, \alpha_{M}\right\rangle$ is not in $x_{q}$. We apply Lemma 7.4 to show that we can add $\left\langle\gamma, \alpha_{M}\right\rangle$ to $q$. This is a contradiction, since any extension of $q$ forces that $\alpha$ is a limit point of $\dot{C}_{S}$.

Since $\langle\gamma, \gamma\rangle \in x_{q}$, it follows that $\left\langle\gamma_{M}, \gamma_{M}\right\rangle \in x_{q}$ since $q$ is a condition. By the maximality of $\gamma, \gamma=\gamma_{M}$. So $\gamma \in M$. Also by the maximality of $\gamma,\left\langle\gamma, \alpha_{M}\right\rangle$ does not overlap any pair in $x_{q}$ of ordinals below $\alpha$. And if $\left\langle\beta, \beta^{\prime}\right\rangle \in x_{q}$ with $\alpha \leq \beta$, then $\alpha_{M}<\beta$ by our case assumption. Hence $\left\langle\gamma, \alpha_{M}\right\rangle$ does not overlap any pair in $x_{q}$.

Now let $N \in A_{q}$ be given such that $N \cap \omega_{2} \nsubseteq \alpha$. We will show that either $\gamma$ and $\alpha_{M}$ are in $N$, or $N \cap\left[\gamma, \alpha_{M}\right]=\emptyset$ and $\left\langle\gamma_{N}, \gamma_{N}\right\rangle \in x_{q}$. Obviously $N \notin A_{0}$. Suppose that $N \in A_{1}$. Then $\left\langle\alpha_{N}, \alpha_{N}\right\rangle \in x_{q}$ as noted above. And by the choice of $\gamma, N \cap \alpha \subseteq \gamma$. So $\gamma_{N}=\alpha_{N}$. Hence $\left\langle\gamma_{N}, \gamma_{N}\right\rangle \in x_{q}$.

Suppose that $N \in A_{2}$. First assume that for all $K \in A_{2}, N \cap \omega_{1} \leq K \cap \omega_{1}$. Then by (i) and (ii), $N \cap \omega_{1}=M \cap \omega_{1}$ and $\alpha_{M} \leq \alpha_{N}$. Since $\alpha<\beta_{M, N}$ and $\gamma \in M \cap \alpha$, $\gamma \in N$. We claim $\alpha_{M}=\alpha_{N}$. If not, then $\beta_{M, N} \leq \alpha_{M}$. But then $\alpha<\beta_{M, N} \leq \alpha_{M}$ implies that $\alpha_{M}=\min \left(M \backslash \beta_{M, N}\right)$. So $\alpha_{M} \in R_{A}$, and therefore $\left\langle\alpha_{M}, \alpha_{M}\right\rangle \in x_{q}$, which contradicts our case assumption. It follows that $\gamma$ and $\alpha_{M}$ are both in $N$.

Now assume that $M<N$. Recall that $\alpha<\beta_{M, N}$. So if $\beta_{M, N} \leq \alpha_{M}$, then $\alpha_{M}=\min \left(M \backslash \beta_{M, N}\right)$ and hence $\alpha_{M} \in R_{N}(M)$. This implies that $\left\langle\alpha_{M}, \alpha_{M}\right\rangle \in x_{q}$, which contradicts our case assumption. So $\alpha_{M}<\beta_{M, N}$. Since $M \cap \beta_{M, N} \subseteq N, \gamma$ and $\alpha_{M}$ are in $N$.

\section{Preserving Cardinals larger than $\omega_{2}$}

In this final section we will prove a general amalgamation result for models of size $\omega_{1}$. As a consequence, we show that the forcing poset consisting of finite coherent adequate sets in $H(\lambda)$ ordered by inclusion is $\omega_{2}$-c.c 2 Although we did not need this fact for the other results in this paper, we record it here for future applications.

Lemma 8.1. Let $M_{0}, M_{1}, K_{0}$, and $K_{1}$ be in $\mathcal{X}$. Let $\beta \in \Lambda$. Assume that $M_{0} \cap \beta=$ $M_{1} \cap \beta, K_{0} \cap \beta=K_{1} \cap \beta, \beta_{K_{0}, M_{0}} \leq \beta$, and $\beta_{K_{1}, M_{1}} \leq \beta$. Then $\beta_{K_{0}, M_{0}}=\beta_{K_{1}, M_{1}}$.

Proof. Clearly $K_{0} \cap \beta=K_{1} \cap \beta$ and $M_{0} \cap \beta=M_{1} \cap \beta$ imply that $\Lambda_{M_{0}} \cap(\beta+1)=$ $\Lambda_{M_{1}} \cap(\beta+1)$ and $\Lambda_{K_{0}} \cap(\beta+1)=\Lambda_{K_{1}} \cap(\beta+1)$. Since $\beta_{K_{0}, M_{0}}$ and $\beta_{K_{1}, M_{1}}$ are both in $\beta+1, \beta_{K_{0}, M_{0}}=\max \left(\Lambda_{K_{0}} \cap \Lambda_{M_{0}}\right)=\max \left(\Lambda_{K_{0}} \cap \Lambda_{K_{1}} \cap(\beta+1)\right)=$ $\max \left(\left(\Lambda_{K_{0}} \cap(\beta+1)\right) \cap\left(\Lambda_{M_{0}} \cap(\beta+1)\right)\right)=\max \left(\left(\Lambda_{K_{1}} \cap(\beta+1)\right) \cap\left(\Lambda_{M_{1}} \cap(\beta+1)\right)\right)=$ $\max \left(\Lambda_{K_{1}} \cap \Lambda_{M_{1}} \cap(\beta+1)\right)=\max \left(\Lambda_{K_{1}} \cap \Lambda_{M_{1}}\right)=\beta_{K_{1}, M_{1}}$.

\footnotetext{
${ }^{2}$ If $2^{\omega_{1}}=\omega_{2}$ and $\lambda^{<\lambda}=\lambda$, then the chain condition follows immediately from the conjunction of Proposition 4.4 of [5] and Lemma 2.5 of [2]. A proof of Lemma 2.5 of [2] can be found in Lemma 3.9 of 1 .
} 
Proposition 8.2. Let $A$ be a finite coherent adequate set. Let $\chi \geq \lambda$ be a regular cardinal. Assume that $N^{*}$ is an elementary substructure of $(H(\chi), \in)$ of size $\omega_{1}$ such that $\beta^{*}:=N^{*} \cap \omega_{2} \in \Lambda$. Let $\beta \in \beta^{*} \cap \Lambda$.

Assume that there exists a map $M \mapsto M^{\prime}$ from $A$ into $N^{*}$ satisfying:

(1) $M \cong M^{\prime}, M \cap \beta^{*}=M^{\prime} \cap \beta$, and $M \cap N^{*} \subseteq M^{\prime}$;

(2) $K \in M$ iff $K^{\prime} \in M^{\prime}$, and in that case, $\sigma_{M, M^{\prime}}(K)=K^{\prime}$;

(3) if $M \in N^{*}$ then $M=M^{\prime}$;

(4) $A^{\prime}:=\left\{M^{\prime}: M \in A\right\}$ is a coherent adequate set.

Then $C:=A \cup A^{\prime}$ is a coherent adequate set. Moreover, if we let $r_{A}=\left\{\min \left(M \backslash \beta^{*}\right)\right.$ : $M \in A\}$ and $r_{A^{\prime}}=\left\{\min \left(M^{\prime} \backslash \beta\right): M \in A\right\}$, then $R_{C} \subseteq R_{A} \cup R_{A^{\prime}} \cup r_{A} \cup r_{A^{\prime}}$.

Proof. For all $M \in A$, since $M \cap \beta^{*}=M^{\prime} \cap \beta, \sigma_{M, M^{\prime}} \uparrow \beta$ is the identity function. Also note that $M \cap \beta^{*}=M^{\prime} \cap \beta$ obviously implies that $M \cap \beta^{*} \subseteq \beta$ and $M \cap \beta=$ $M^{\prime} \cap \beta$.

First we will prove that $C$ is adequate and that the remainder points of $C$ are as required. Since $A$ and $A^{\prime}$ are each adequate, we only need to compare models in $A$ with models in $A^{\prime}$. So let $K$ and $M$ be in $A$ and we will compare $K$ and $M^{\prime}$. Since $M^{\prime} \in N^{*}, M^{\prime} \cap \omega_{2} \subseteq \beta^{*}$. So $\beta_{K, M^{\prime}} \leq \beta^{*}$ by Lemma 1.7(1). If $\beta<\beta_{K, M^{\prime}}$ then by Lemma $1.2, K \cap\left[\beta, \beta_{K, M^{\prime}}\right)$ is nonempty. But then $K \cap\left[\beta, \beta^{*}\right)$ is nonempty, which is false. So $\beta_{K, M^{\prime}} \leq \beta$.

We claim that either $\beta^{*} \leq \beta_{K, M}$ or $\beta_{K, M}=\beta_{K, M^{\prime}}$. In particular, $\beta_{K, M^{\prime}} \leq \beta_{K, M}$. For suppose that $\beta_{K, M}<\beta^{*}$. If $\beta<\beta_{K, M}$, then by Lemma 1.7(2), there is $\xi \in K \cap M \cap\left[\beta, \beta_{K, M}\right)$. But then $\xi \in K \cap \beta^{*} \subseteq \beta$, which is a contradiction. So $\beta_{K, M} \leq \beta$. Now apply Lemma 8.1 letting $K_{0}=K_{1}=K, M_{0}=M$, and $M_{1}=M^{\prime}$. Then obviously $K_{0} \cap \beta=K_{1} \cap \beta, M_{0} \cap \beta=M_{1} \cap \beta, \beta_{K_{0}, M_{0}}=\beta_{K, M} \leq \beta$, and $\beta_{K_{1}, M_{1}}=\beta_{K, M^{\prime}} \leq \beta$. So by Lemma 8.1, $\beta_{K, M}=\beta_{K, M^{\prime}}$.

Now we show that $\left\{K, M^{\prime}\right\}$ is adequate.

Case 1: $K<M$. Then $K \cap \beta_{K, M} \in M$. Since $\beta_{K, M^{\prime}} \leq \beta_{K, M}, K \cap \beta_{K, M^{\prime}} \in M$. As $\beta_{K, M^{\prime}} \leq \beta$ and $K \cap \beta=K^{\prime} \cap \beta, K \cap \beta_{K, M^{\prime}}=K^{\prime} \cap \beta_{K, M^{\prime}}$. Since $K^{\prime} \in N^{*}$, $K \cap \beta_{K, M^{\prime}} \in M \cap N^{*}$. But $M \cap N^{*} \subseteq M^{\prime}$. So $K \cap \beta_{K, M^{\prime}} \in M^{\prime}$, and therefore $K<M^{\prime}$.

Case 2: $K \sim M$. Since $\beta_{K, M^{\prime}} \leq \beta_{K, M}, K \cap \beta_{K, M^{\prime}}=M \cap \beta_{K, M^{\prime}}$. As $\beta_{K, M^{\prime}} \leq \beta$ and $M \cap \beta=M^{\prime} \cap \beta, M \cap \beta_{K, M^{\prime}}=M^{\prime} \cap \beta_{K, M^{\prime}}$. So $K \cap \beta_{K, M^{\prime}}=M^{\prime} \cap \beta_{K, M^{\prime}}$.

Case 3: $M<K$. Then $M \cap \beta_{K, M} \in K$. Since $\beta_{K, M^{\prime}} \leq \beta_{K, M}, M \cap \beta_{K, M^{\prime}} \in K$. But $\beta_{K, M^{\prime}} \leq \beta$ and $M \cap \beta=M^{\prime} \cap \beta$. So $M \cap \beta_{K, M^{\prime}}=M^{\prime} \cap \beta_{K, M^{\prime}}$. Therefore $M^{\prime} \cap \beta_{K, M^{\prime}} \in K$. Hence $M^{\prime}<K$.

Now we show that the remainder points are as required.

Let $\zeta \in R_{K}\left(M^{\prime}\right)$ be given. First assume that $\zeta \geq \beta$. If $\zeta=\min \left(M^{\prime} \backslash \beta_{K, M^{\prime}}\right)$, then since $\beta_{K, M^{\prime}} \leq \beta, \zeta=\min \left(M^{\prime} \backslash \zeta\right)$. So $\zeta \in r_{A^{\prime}}$ and we are done. Otherwise $\zeta=\min \left(M^{\prime} \backslash \gamma\right)$ for some $\gamma \in K \backslash \beta_{K, M^{\prime}}$. Since $M^{\prime} \cap \omega_{2} \subseteq \beta^{*}, \zeta<\beta^{*}$. As $K \cap \beta^{*} \subseteq \beta, \gamma<\beta$. So again $\zeta=\min \left(M^{\prime} \backslash \beta\right)$ and $\zeta \in r_{A^{\prime}}$.

Secondly, assume that $\zeta<\beta$. Then $\zeta \in M^{\prime} \cap \beta=M \cap \beta$. Since $\beta_{K, M^{\prime}} \leq \zeta$, $\zeta$ is not in $K$. Suppose that $M \leq K$. Since $\zeta \in M \backslash K, \beta_{K, M} \leq \zeta$. As $\zeta<\beta$, $\beta_{K, M}<\beta$. Therefore by the claim above, $\beta_{K, M}=\beta_{K, M^{\prime}}$. If $\zeta=\min \left(M^{\prime} \backslash \beta_{K, M^{\prime}}\right)$, 
then clearly $\zeta=\min \left(M \backslash \beta_{K, M}\right)$ and $\zeta \in R_{A}$. Suppose that $\zeta=\min \left(M^{\prime} \backslash \gamma\right)$ for some $\gamma \in K \backslash \beta_{K, M^{\prime}}$. Then $\gamma \in K \backslash \beta_{K, M}$ and $\zeta=\min (M \backslash \gamma)$, so $\zeta \in R_{A}$.

Now assume that $K<M$. Then $\zeta=\min \left(M^{\prime} \backslash \gamma\right)$ for some $\gamma \in K \backslash \beta_{K, M^{\prime}}$. Since $\zeta<\beta, \gamma<\beta$. Hence $\gamma$ is not in $M$ since otherwise it would be in $M^{\prime}$. So $\gamma \in K \backslash M$. Since $K<M$, this implies that $\beta_{K, M} \leq \gamma$. So $\beta_{K, M}=\beta_{K, M^{\prime}}$ by the claim above. Therefore $\gamma \in K \backslash \beta_{K, M}$ and $\zeta=\min (M \backslash \gamma)$. Hence $\zeta$ is in $R_{A}$.

Now consider $\zeta \in R_{M^{\prime}}(K)$. First assume that $\beta \leq \zeta$. Since $K \cap \beta^{*} \subseteq \beta, \beta^{*} \leq \zeta$. But $\beta_{K, M^{\prime}}$ and any ordinal in $M^{\prime} \cap \omega_{2}$ are below $\beta^{*}$. So clearly $\zeta=\min \left(K \backslash \beta^{*}\right)$ and $\zeta \in r_{A}$.

Now suppose that $\zeta<\beta$. First assume that $K \leq M$. Since $\zeta \notin M^{\prime}$ and $M^{\prime} \cap \beta=M \cap \beta, \zeta \notin M$. So $\zeta \in K \backslash M$. Since $K \leq M$, this implies that $\beta_{K, M} \leq \zeta$. Therefore $\beta_{K, M}<\beta$ and hence $\beta_{K, M}=\beta_{K, M^{\prime}}$ by the claim above. If $\zeta=\min \left(K \backslash \beta_{K, M^{\prime}}\right)$, then $\zeta=\min \left(K \backslash \beta_{K, M}\right)$ and therefore $\zeta \in R_{A}$. Otherwise $\zeta=\min (K \backslash \gamma)$ for some $\gamma \in M^{\prime} \backslash \beta_{K, M^{\prime}}$. Since $\zeta<\beta, \gamma<\beta$. So $\gamma \in M^{\prime} \cap \beta=M \cap \beta$. Hence $\gamma \in M \backslash \beta_{K, M}$, so $\zeta \in R_{A}$.

Next assume that $M<K$. Then $\zeta=\min (K \backslash \gamma)$ for some $\gamma \in M^{\prime} \backslash \beta_{K, M^{\prime}}$. Since $\zeta<\beta, \gamma \in M^{\prime} \cap \beta=M \cap \beta$. So $\gamma \in M$. As $\gamma \in M \backslash K$ and $M<K, \beta_{K, M} \leq \gamma<\beta$. By the claim above, $\beta_{K, M}=\beta_{K, M^{\prime}}$. Hence $\gamma \in M \backslash \beta_{K, M}$, so $\zeta \in R_{A}$.

Next we will prove that $C$ is coherent. We verify the conditions (1), (2), and (3) of Definition 1.15.

(1) Let $K$ and $M$ be in $A$ and assume that $K \sim M^{\prime}$. Then $K \cap \omega_{1}=M^{\prime} \cap \omega_{1}=$ $M \cap \omega_{1}$, so $K$ and $M$ are strongly isomorphic. Since $M$ and $M^{\prime}$ are isomorphic, $K$ and $M^{\prime}$ are isomorphic. To see that $K$ and $M^{\prime}$ are strongly isomorphic, let $a \in K \cap M^{\prime}$ be given. Since $a \in M^{\prime}$ and $M^{\prime} \in N^{*}, a \in K \cap N^{*}$. Since $K \cap N^{*} \subseteq K^{\prime}$, $a \in K^{\prime}$. So $a \in K \cap K^{\prime}$, which implies that $\sigma_{K, K^{\prime}}(a)=a$ since $K$ and $K^{\prime}$ are strongly isomorphic by assumption. Also $a \in K^{\prime} \cap M^{\prime}$, so $\sigma_{K^{\prime}, M^{\prime}}(a)=a$ since $K^{\prime}$ and $M^{\prime}$ are strongly isomorphic. So $\sigma_{K, M^{\prime}}(a)=\sigma_{K^{\prime}, M^{\prime}}\left(\sigma_{K, K^{\prime}}(a)\right)=\sigma_{K^{\prime}, M^{\prime}}(a)=a$.

(2) Let $K$ and $M$ be in $A$. First assume that $K<M^{\prime}$, and we will find $M^{*}$ in $C$ isomorphic to $M^{\prime}$ such that $K \in M^{*}$. Since $K \cap \omega_{1}<M^{\prime} \cap \omega_{1}=M \cap \omega_{1}, K<M$. By the coherence of $A$, there is $M^{*}$ in $A$ isomorphic to $M$ such that $K \in M^{*}$. Then $M^{*}$ is isomorphic to $M^{\prime}$ and we are done.

Now assume that $M^{\prime}<K$, and we will find $K^{*}$ in $C$ isomorphic to $K$ such that $M^{\prime} \in K^{*}$. We have that $M^{\prime} \cap \omega_{1}<K \cap \omega_{1}=K^{\prime} \cap \omega_{1}$, so $M^{\prime}<K^{\prime}$. Since $A^{\prime}$ is coherent, there is $K^{*}$ in $A^{\prime}$ isomorphic to $K^{\prime}$ such that $M^{\prime} \in K^{*}$. Then $K^{*}$ is isomorphic to $K$.

(3) Let $M, N$, and $K$ be in $C$, where $M$ and $N$ are isomorphic and $K \in M$. We will prove that $\sigma_{M, N}(K) \in C$.

We claim that if $M$ is in $A$ then so is $K$. So assume that $K=L^{\prime}$ for some $L \in A$. Since $L^{\prime} \in M \cap N^{*}$ and $M \cap N^{*} \subseteq M^{\prime}, L^{\prime} \in M \cap M^{\prime}$. Therefore $\sigma_{M, M^{\prime}}\left(L^{\prime}\right)=L^{\prime}$. But $L^{\prime} \in M^{\prime}$ implies that $L \in M$ and $\sigma_{M, M^{\prime}}(L)=L^{\prime}$. Hence $L=L^{\prime}$ and therefore $L^{\prime}=K$ is in $A$.

Case 1: $M$ and $N$ are in $A$. Then $K \in A$, so we are done since $A$ is coherent. 
Case 2: $M$ is in $A$ and $N=P^{\prime}$ for some $P \in A$. Then again $K$ is in $A$. Let $Q=\sigma_{M, P}(K)$, which is in $A$ since $A$ is coherent. Then $\sigma_{P, P^{\prime}}(Q)=Q^{\prime}$ is in $A^{\prime}$. And $\sigma_{M, P^{\prime}}(K)=\sigma_{P, P^{\prime}}\left(\sigma_{M, P}(K)\right)=\sigma_{P, P^{\prime}}(Q)=Q^{\prime}$ which is in $A^{\prime}$ and hence in $C$.

Case 3: $M=M_{0}^{\prime}$ and $N=N_{0}^{\prime}$ for some $M_{0}$ and $N_{0}$ in $A$. If $K=L^{\prime}$ for some $L \in A$, then we are done since $A^{\prime}$ is coherent. Suppose that $K \in A$. Then $K \in M_{0}^{\prime}$ and $M_{0}^{\prime} \in N^{*}$, which imply that $K \in N^{*}$. So $K=K^{\prime}$. Hence $K \in A^{\prime}$ so $\sigma_{M, N}(K) \in A^{\prime}$ since $A^{\prime}$ is coherent.

Theorem 8.3. Assume $\mathrm{CH}$. Then the forcing poset consisting of finite coherent adequate sets ordered by inclusion is $\omega_{2}$-c.c.

Proof. Let $\mathcal{A}$ be a maximal antichain, and we will prove that $|\mathcal{A}| \leq \omega_{1}$. Fix a regular cardinal $\chi>\lambda$ such that $\mathbb{P}$ is in $H(\lambda)$. By CH, we can fix $N^{*}$ an elementary substructure of $H(\chi)$ of size $\omega_{1}$ such that $Y, \mathbb{P}$, and $\mathcal{A}$ are in $N^{*}$ and $\left(N^{*}\right)^{\omega} \subseteq N^{*}$.

Since $N^{*}$ has size $\omega_{1}$, we will be done if we can show that $\mathcal{A} \subseteq N^{*}$. Let $\beta^{*}:=$ $N^{*} \cap \omega_{2}$. Note that $\beta^{*}$ is in $\Lambda$. And by elementarity, $\Lambda \cap \beta^{*}$ is cofinal in $\beta^{*}$.

Let $A=\left\{M_{0}, \ldots, M_{k}\right\}$ be a condition in $\mathcal{A}$. Fix $\beta \in \Lambda \cap N^{*}$ large enough so that for all $M \in A, \sup \left(M \cap \beta^{*}\right)<\beta$. Let $R$ be the relation where $R(i, j)$ holds if $M_{i} \in M_{j}$. Let $d$ be the set of $i \leq k$ such that $M_{i} \in N^{*}$.

For each $i \leq k$ let $\mathfrak{M}_{i}$ denote the structure $\left(M_{i}, \in, Y \cap M_{i}\right)$, and let $\overline{\mathfrak{M}}_{i}$ denote its transitive collapse. Recall that $\sigma_{M_{i}}$ is the transitive collapsing map of $\mathfrak{M}_{i}$. For each pair $\langle i, j\rangle$ in $R$, let $J_{i, j}:=\sigma_{M_{j}}\left(M_{i}\right)$. For each $i \leq k$ let $S_{i}$ be the relation where $S_{i}(a, b)$ holds if $a \in M_{i} \cap N^{*}$ and $\sigma_{M_{i}}(a)=b$.

Since $R$ and $d$ are finite, they are in $N^{*}$. By CH, $H\left(\omega_{1}\right) \subseteq N^{*}$. So each transitive collapse $\overline{\mathfrak{M}}_{i}$ and each object $J_{i, j}$ is in $N^{*}$. As $N^{*}$ is countably closed, each set $M_{i} \cap N^{*}$ is a member of $N^{*}$. So each relation $S_{i}$ is in $N^{*}$.

The objects $M_{0}, \ldots, M_{k}$ witness in the model $H(\chi)$ that there exist $M_{0}^{\prime}, \ldots, M_{k}^{\prime}$ satisfying:

(a) $\left\{M_{0}^{\prime}, \ldots, M_{k}^{\prime}\right\}$ is a finite coherent adequate set in $\mathcal{A}$;

(b) for $i \leq k, M_{i}^{\prime} \cap \beta=M_{i} \cap \beta^{*}, M_{i} \cap N^{*} \subseteq M_{i}^{\prime}$, and the transitive collapse of the structure $\left(M_{i}^{\prime}, \in, Y \cap M_{i}^{\prime}\right)$ is equal to $\overline{\mathfrak{M}}_{i}$;

(c) $R(i, j)$ iff $M_{i}^{\prime} \in M_{j}^{\prime}$, and in that case, $\sigma_{M_{j}^{\prime}}\left(M_{i}^{\prime}\right)=J_{i, j}$;

(d) for $i \leq k$, if $S_{i}(a, b)$ then $\sigma_{M_{i}^{\prime}}(a)=b$;

(e) for $i \in d, M_{i}=M_{i}^{\prime}$.

The parameters mentioned in the above statement are those described in the previous paragraph together with $\mathcal{A}, \beta, M_{i} \cap \beta^{*}$ for $i \leq k$, and $M_{i}$ for $i \in d$. So the parameters are all members of $N^{*}$. By the elementarity of $N^{*}$, fix $M_{0}^{\prime}, \ldots, M_{k}^{\prime}$ in $N^{*}$ satisfying the same statement.

Let us show that the map $M_{i} \mapsto M_{i}^{\prime}$ for $i \leq k$ satisfies the assumptions (1)-(4) of Proposition 8.2. By (a), $\left\{M_{0}^{\prime}, \ldots, M_{k}^{\prime}\right\}$ is a coherent adequate set, so (4) holds. For (3), if $M_{i} \in N^{*}$, then $i \in d$, and so by (e), $M_{i}=M_{i}^{\prime}$.

(1) Consider $i \leq k$. By (b), $M_{i} \cap \beta^{*}=M_{i}^{\prime} \cap \beta$ and $M_{i} \cap N^{*} \subseteq M_{i}^{\prime}$. Also by (b), the structures $\left(M_{i}, \in, Y \cap M_{i}\right)$ and $\left(M_{i}^{\prime}, \in, Y \cap M_{i}^{\prime}\right)$ have the same transitive collapse and hence are isomorphic. To see that $M_{i} \cong M_{i}^{\prime}$, let $a \in M_{i} \cap M_{i}^{\prime}$. Then $a \in M_{i} \cap N^{*}$. Let $b:=\sigma_{M_{i}}(a)$. Then $S_{i}(a, b)$ holds. By $(\mathrm{d}), \sigma_{M_{i}^{\prime}}(a)=b$. Hence $\sigma_{M_{i}, M_{i}^{\prime}}(a)=\sigma_{M_{i}^{\prime}}^{-1}\left(\sigma_{M_{i}}(a)\right)=\sigma_{M_{i}^{\prime}}^{-1}(b)=a$. 
(2) By the definition of $R$ and (c), $M_{i} \in M_{j}$ iff $R(i, j)$ iff $M_{i}^{\prime} \in M_{j}^{\prime}$. And in that case, $\sigma_{M_{j}^{\prime}}\left(M_{i}^{\prime}\right)=J_{i, j}$. Now $J_{i, j}=\sigma_{M_{j}}\left(M_{i}\right)$ by definition. So $\sigma_{M_{j}, M_{j}^{\prime}}\left(M_{i}\right)=$ $\sigma_{M_{j}^{\prime}}^{-1}\left(\sigma_{M_{j}}\left(M_{i}\right)\right)=\sigma_{M_{j}^{\prime}}^{-1}\left(J_{i, j}\right)=M_{i}^{\prime}$.

This completes the verification of the assumptions of Proposition 8.2. Let $C:=$ $A \cup A^{\prime}$. Then $C$ is a finite coherent adequate set, and obviously $C \leq A, A^{\prime}$. Hence $A$ and $A^{\prime}$ are compatible. But $A$ and $A^{\prime}$ are both in $\mathcal{A}$. Since $\mathcal{A}$ is an antichain, $A=A^{\prime}$. Therefore $A \in N^{*}$. Thus we have shown that $\mathcal{A} \subseteq N^{*}$, completing the proof.

By a somewhat easier argument, if $\mathrm{CH}$ fails then the forcing poset consisting of finite coherent adequate sets ordered by inclusion satisfies the $\left(2^{\omega}\right)^{+}$-c.c. Hence by the material in Section 3, if $2^{\omega}<\lambda$ then this forcing poset collapses $2^{\omega}$ to have size $\omega_{1}$, forces $\mathrm{CH}$, and preserves all cardinals above $2^{\omega}$.

\section{REFERENCES}

[1] D. Asperó and M.A. Mota. Forcing consequences of PFA together with the continuum large. To appear in Trans. Amer. Math. Soc.

[2] D. Asperó and M.A. Mota. Separating club-guessing principles in the presence of fat forcing axioms. Submitted.

[3] S.D. Friedman. Forcing with finite conditions. In Set Theory: Centre de Recerca Matemàtica, Barcelona, 2003-2004, Trends in Mathematics, pages 285-295. Birkhäuser Verlag, 2006.

[4] J. Krueger. Adding a club with finite conditions, part II. To appear in Archive for Mathematical Logic.

[5] J. Krueger. Forcing with adequate sets of models as side conditions. Submitted.

[6] J. Krueger. Coherent adequate sets and forcing square. Fund. Math., 224:279-300, 2014.

[7] W. Mitchell. $I\left[\omega_{2}\right]$ can be the nonstationary ideal on Cof $\left(\omega_{1}\right)$. Trans. Amer. Math. Soc., 361(2):561601, 2009.

[8] T. Miyamoto. Forcing a quagmire via matrices of models. Preprint.

[9] I. Neeman. Forcing with sequences of models of two types. Notre Dame J. Form. Log., 55(2):265-298, 2014.

[10] S. Todorčević. A note on the proper forcing axiom. In Axiomatic set theory (Boulder, Colo., 1983), volume 31 of Contemp. Math., pages 209-218. Amer. Math. Soc., Providence, RI, 1984.

John Krueger, Department of Mathematics, University of North Texas, 1155 Union Circle \#311430, Denton, TX 76203

E-mail address: jkrueger@unt.edu

Miguel Angel Mota, Department of Mathematics, University of Toronto, Toronto, Ontario, Canada M5S 2E4

E-mail address: motagaytan@gmail.com 